\title{
Lazy groupoids
}

\section{Balázs Kaprinai $^{1} \cdot$ Hajime Machida ${ }^{2} \cdot$ Tamás Waldhauser $^{1}$}

Received: 2 May 2020 / Accepted: 8 June 2020 / Published online: 20 October 2020

(c) The Author(s) 2020

\begin{abstract}
A binary operation $f(x, y)$ is said to be lazy if every operation that can be obtained from $f$ by composition is equivalent to $f(x, y), f(x, x)$ or $x$. We describe lazy operations by identities (i.e., we determine all varieties of lazy groupoids), and we also characterize lazy groupoids up to isomorphism.
\end{abstract}

Keywords Binary operation · Groupoid · Semigroup · Term operation · Clone · Variety $\cdot$ Lazy operation $\cdot$ Lazy groupoid

\section{Introduction}

Given a (not necessarily associative) binary operation $f(x, y)=x y$, we can form many other operations by composing $f$ by itself, such as $(x y) z,((x y)(z u))(u(y v))$, $x_{1}\left(x_{2}\left(x_{3} \cdots\left(x_{n-1} x_{n}\right)\right)\right)$, etc. These composite operations can have arbitrarily many variables, but sometimes it happens that they do not depend on all of their variables. Consider, for example, a rectangular band, i.e., a semigroup satisfying the identities $x x \approx x$ (idempotency) and $x y z \approx x z$. These identities imply $x_{1} x_{2} \cdots x_{n} \approx x_{1} x_{n}$ for all $n \in \mathbb{N}$, thus every product can be reduced to a product of at most two variables. It is natural to say that the multiplication of a rectangular band is lazy, since it only generates the operations $f(x, y)$ and $f(x, x)$ (up to renaming variables), and we can get these from $f$ by simply identifying variables, hence composition is "unproductive" in this case.

Communicated by Victoria Gould.

Tamás Waldhauser

twaldha@math.u-szeged.hu

Balázs Kaprinai

kaprinaibali@gmail.com

Hajime Machida

machida.zauber@gmail.com

1 Bolyai Institute, University of Szeged, Aradi vértanúk tere 1, Szeged 6720, Hungary

2 Tokyo, Japan 
Motivated by this example, we shall say that a binary operation $f$ on a set $A$ is lazy, if the only operations that can be obtained from $f$ by composition are $f(x, y)$ and $f(x, x)$. We will give a more precise definition of laziness for operations of arbitrary arities in the Sect. 2. The main goal of this paper is to describe all lazy binary operations and the corresponding groupoids $(A ; f)$. In Sect. 3 we will characterize lazy groupoids by identities: we will prove that they fall into 15 varieties (Theorem 3.5). One of these varieties is the semigroup variety defined by $(x y) z \approx x(y z) \approx x z$, which contains rectangular bands as a subvariety. We will determine all subvarieties of the 15 maximal lazy groupoid varieties in Sect. 4 (Theorem 4.2).

In Sect. 5 we give a more concrete description of lazy groupoids: we characterize them up to isomorphism by explicitly constructing their multiplication tables. This description is similar in spirit to the well known construction of rectangular bands as groupoids of the form $\left(A_{1} \times A_{2} ; \cdot\right)$, where the multiplication is defined by $\left(a_{1}, a_{2}\right) \cdot\left(b_{1}, b_{2}\right)=\left(a_{1}, b_{2}\right)$.

Lazy operations were originally defined in [5] in connection with essentially minimal clones. The 15 varieties of lazy groupoids were described already in the conference paper [6] (but the proof of Theorem 3.5 was only sketched there), and then an application to essentially minimal clones was given. Thus, the present paper can be regarded as an extended version of [6], the new contributions being the determination of all (sub)varieties of lazy groupoids and the explicit description of lazy groupoids up to isomorphism.

\section{Preliminaries}

An $n$-ary operation on a nonempty set $A$ is a map $f: A^{n} \rightarrow A$. We denote the set of $n$-ary operations on $A$ by $\mathcal{O}_{A}^{(n)}$, and $\mathcal{O}_{A}$ stands for the set of all finitary operations on $A$. We say that the $i$-th variable of $f \in \mathcal{O}_{A}^{(n)}$ is essential (in other words, $f$ depends on its $i$-th variable) if there exist $a_{1}, \ldots, a_{i}, a_{i}^{\prime}, \ldots, a_{n} \in A$ such that

$$
f\left(a_{1}, \ldots, a_{i}, \ldots, a_{n}\right) \neq f\left(a_{1}, \ldots, a_{i}^{\prime}, \ldots, a_{n}\right) .
$$

For $1 \leq i \leq n \in \mathbb{N}$ we define the $i$-th $n$-ary projection $e_{i}^{(n)} \in \mathcal{O}_{A}^{(n)}$ by $e_{i}^{(n)}\left(x_{1}, \ldots, x_{n}\right)=x_{i}$. The set of all projections on $A$ is denoted by $\mathcal{J}_{A}$. Observe that $e_{1}^{(1)}=\mathrm{id}$ is the identity function on $A$.

A clone is a set $\mathcal{C} \subseteq \mathcal{O}_{A}$ of operations that is closed under composition and contains every projection. The clone generated by a given operation $f$ is the clone $[f]$ containing all operations that can be obtained from $f$ and the projections by composition. Equivalently, $[f]$ is the clone of term functions of the algebra $\mathbb{A}=(A ; f)$. If $f$ is a binary operation, then we will use the notation $f(x, y)=x \cdot y=x y$, and then the algebra $\mathbb{A}=(A ; f)=(A ; \cdot)$ is called a groupoid. For the sake of simplicity, let us say that the groupoid $\mathbb{A}$ is essentially binary (essentially at most unary) if $f$ depends on both of its variables ( $f$ depends on at most one variable).

For $f \in \mathcal{O}_{A}^{(n)}$ and $g \in \mathcal{O}_{A}^{(m)}$, we say that $g$ is an identification minor (or simply a minor) of $f$ (notation: $g \preceq f$ ), if there exists a map $\sigma:\{1,2, \ldots, n\} \rightarrow\{1,2, \ldots, m\}$ such that 


$$
g\left(x_{1}, \ldots, x_{m}\right)=f\left(x_{\sigma(1)}, \ldots, x_{\sigma(n)}\right) .
$$

This means that $g$ can be obtained from $f$ by identifying variables, permuting variables and introducing inessential variables. The relation $\preceq$ gives rise to a quasiorder on $\mathcal{O}_{A}$. The corresponding equivalence relation is defined by $f \equiv g \Longleftrightarrow f \preceq g$ and $g \preceq f$, and it is clear that $f \equiv g$ if and only if they differ only in inessential variables and/or in the order of their variables. Note that for any $f \in \mathcal{O}_{A}$, we have $f \equiv$ id if and only if $f \in \mathcal{J}_{A}$. We use the notation $\downarrow f$ for the principal ideal (downset) generated by $f$ in the subfunction quasiorder: $\downarrow f:=\left\{g \in \mathcal{O}_{A}: g \preceq f\right\}$. Note that the set $\downarrow f$ contains only one unary operation, namely $f(x, \ldots, x)$. For more information on the minor quasiorder and its principal ideals, see [4].

Clearly, $\mathcal{J}_{A} \cup \downarrow f \subseteq[f]$ holds for every operation $f$. If $[f]=\mathcal{J}_{A} \cup \downarrow f$, then we say that $f$ is a lazy operation and $[f]$ is a lazy clone. Thus $f$ is a lazy operation if it does not generate any other operations but its identification minors and projections.

Example 2.1 A unary operation $f \in \mathcal{O}_{A}^{(1)}$ is lazy if and only if the algebra $(A ; f)$ satisfies $f^{2}(x) \approx x$ or $f^{2}(x) \approx f(x)$ (where $f^{2}(x)$ stands for $f(f(x))$ ). Indeed, if $f$ is lazy, then $f^{2} \in \mathcal{J}_{A} \cup \downarrow f$, and the latter set contains only two operations up to equivalence, namely id and $f$. Thus we have $f^{2} \equiv * i d$ (hence $f^{2}(x) \approx x$ ) or $f^{2} \equiv f$ (hence $\left.f^{2}(x) \approx f(x)\right)$. Conversely, each one of the given identities implies that $[f]=\{\mathrm{id}, f\}$, and thus $f$ is lazy.

Example 2.2 If $f$ is idempotent, i.e., $f(x, \ldots, x) \approx x$, then $\mathcal{J}_{A} \subseteq \downarrow f$, hence in this case laziness is equivalent to $[f]=\downarrow f$. Lazy idempotent operations can be constructed as follows. Let $A_{1}, \ldots, A_{n}$ be nonempty sets, and let us define an $n$-ary operation $f$ on $A_{1} \times \cdots \times A_{n}$ by

$$
f\left(\left(a_{1}^{1}, \ldots, a_{n}^{1}\right), \ldots,\left(a_{1}^{n}, \ldots, a_{n}^{n}\right)\right)=\left(a_{1}^{1}, \ldots, a_{n}^{n}\right)
$$

for all $a_{i}^{j} \in A_{i}(i, j=1,2, \ldots, n)$. Note that the algebra $\left(A_{1} \times \cdots \times A_{n} ; f\right)$ is the direct product of the algebras $\left(A_{i} ; e_{i}^{(n)}\right)(i=1,2, \ldots, n)$. These algebras were called $n$-dimensional diagonal algebras in [7] and $n$-ary rectangular bands in [6] (cf. the construction of binary rectangular bands in Sect. 1). It was shown in [6, 7] that an idempotent operation $f \in \mathcal{O}_{A}^{(n)}$ is lazy if and only if $(A ; f)$ is (isomorphic to) an $n$-ary rectangular band.

Remark 2.3 For $f \in \mathcal{O}_{A}^{(n)}$ and $k \in\{1,2, \ldots, n\}$, let $f_{k} \in \mathcal{O}_{A}^{(2 n-1)}$ denote the function obtained from $f$ by substituting $f$ for its $k$-th variable; more precisely,

$$
f_{k}\left(x_{1}, \ldots, x_{2 n-1}\right):=f\left(x_{1}, \ldots, x_{k-1}, f\left(x_{k}, \ldots, x_{k+n-1}\right), x_{k+n}, \ldots, x_{2 n-1}\right) .
$$

Clearly, if the operation $f \in \mathcal{O}_{A}^{(n)}$ is lazy then $f_{k} \preceq f$ or $f_{k} \in \mathcal{J}_{A}$ for every $k \in\{1,2, \ldots, n\}$. This is a simple necessary condition for laziness, and it will serve as a starting point for our investigation of lazy binary operations. Note, however, that this condition is not sufficient for laziness, as it is shown by the following example. Let us consider the binary operation $f(x, y)=x y$ on the set $A=\{0,1,2\}$ that is defined by the multiplication table below. 


$$
\begin{array}{l|lll} 
& 0 & 1 & 2 \\
\hline 0 & 1 & 1 & 0 \\
1 & 1 & 1 & 0 \\
2 & 0 & 0 & 0
\end{array}
$$

One can verify that this groupoid satisfies the identities $(x y) z \approx z^{2}$ and $x(y z) \approx x^{2}$, which means that the functions $f_{1}(x, y)=(x y) z$ and $f_{2}(x, y)=x(y z)$ are both minors of $f$. However, $f$ is not a lazy operation, because $g(x, y, z, u):=(x y)(z u)$ is constant 1 , and $g \notin \mathcal{J}_{A} \cup \downarrow f$.

\section{Characterizing lazy groupoids by identities}

Let $f(x, y)=x \cdot y=x y$ denote a binary operation on an arbitrary nonempty set $A$, and let $\mathbb{A}$ denote the groupoid $(A ; f)=(A ; \cdot)$. The dual of $\mathbb{A}$ is the groupoid $\mathbb{A}^{d}=(A ; g)$, where $g(x, y)=y x$, and the dual of a groupoid variety $V$ is $V^{d}=\left\{\mathbb{A}^{d}: \mathbb{A} \in V\right\}$. Clearly, a groupoid is lazy if and only if its dual is lazy.

If $f$ depends only on at most one variable, then we have either $f(x, y)=g(x)$ or $f(x, y)=g(y)$ for some unary operation $g$. According to Example 2.1, $f$ is lazy if and only if $g$ satisfies either $g(g(x)) \approx g(x)$ or $g(g(x)) \approx x$. This yields the following description of essentially at most unary lazy groupoids.

Lemma 3.1 If $\mathbb{A}$ is a lazy essentially at most unary groupoid, then $\mathbb{A}$ belongs to one of the following four varieties:

$$
\begin{array}{ll}
U: x y \approx x z,(x y) z \approx x y ; & U^{d}: x y \approx z y, x(y z) \approx y z ; \\
\widetilde{U}: x y \approx x z,(x y) z \approx x ; & \widetilde{U}^{d}: x y \approx z y, x(y z) \approx z .
\end{array}
$$

Proof The fact that $f$ does not depend on its second variable can be expressed by the identity $x y \approx x z$. Using the unary operation $g$ as above, $g(g(x)) \approx g(x)$ translates to $(x y) z \approx x y$ and $g(g(x)) \approx x$ translates to $(x y) z \approx x$, yielding the varieties $U$ and $\widetilde{U}$. If $f$ does not depend on its first variable, then we obtain the dual varieties $U^{d}$ and $\widetilde{U}^{d}$.

In the sequel, we will assume that $f$ depends on both of its variables. We have $f_{1}(x, y)=(x y) z$ and $f_{2}(x, y)=x(y z)$; see Remark 2.3. If $f$ is a lazy operation then $f_{1}, f_{2} \in \mathcal{J}_{A} \cup \downarrow f$, hence $\mathbb{A}$ satisfies the identities $(x y) z \approx t_{1}$ and $x(y z) \approx t_{2}$ for some choice of the terms $t_{1}, t_{2} \in\left\{x, y, z, x^{2}, y^{2}, z^{2}, x y, y x, y z, z y, x z, z x\right\}$. This gives us 144 possibilities; we will prove that only 20 of these are possible. Examining these cases, we will find that essentially binary lazy groupoids belong to 13 varieties, each being defined by two identities.

Lemma 3.2 If the binary operation $f(x, y)=x y$ satisfies the identity $(x y) z \approx t_{1}$ for some $t_{1} \in\{x, y, z, z y, z x, y x\}$, or it satisfies $x(y z) \approx t_{2}$ for some $t_{2} \in\{z, y, x, y x, z x, z y\}$, then $f$ is essentially at most unary. 
Proof The identity $(x y) z \approx t_{1}(x, y, z)$ implies

$$
t_{1}(x y, z, u) \approx((x y) z) u \approx t_{1}(x, y, z) \cdot u .
$$

If $t_{1}=x$, then we obtain $x y \approx x u$, which shows that $x y$ does not depend on $y$. If $t_{1}=z y$, then we get $u z \approx(z y) u$, which yields $u z \approx u y$ after applying $(z y) u \approx t_{1}(z, y, u)$, hence $f$ does not depend on its second variable.

Let us now consider the case $t_{1}=y x$. Then we have

$$
z(x y) \approx t_{1}(x y, z, u) \approx((x y) z) u \approx t_{1}(x, y, z) \cdot u \approx(y x) u \approx t_{1}(y, x, u) \approx x y,
$$

which immediately implies $(v w)(x y) \approx x y$. On the other hand, we have $(v w)(x y) \approx t_{1}(v, w, x y) \approx w v$. Thus $x y \approx w v$, i.e., $f$ is a constant operation.

Similar arguments work for the remaining three cases; we summarize them in Table 1 of "Appendix 1". The identities $x(y z) \approx t_{2}$ are the duals of the above ones.

Now we are left with 36 pairs $\left(t_{1}, t_{2}\right)$; these possibilities are summarized in Table 2. We will prove in the next two lemmas that the entries marked by '-' contradict the assumption that $f$ is essentially binary, while the other cases give rise to 7 varieties $L_{1}, \ldots, L_{7}$ of lazy groupoids together with their duals (note that $L_{7}$ is selfdual).

Lemma 3.3 Let $\mathbb{A}$ be an essentially binary groupoid. If $\mathbb{A}$ is lazy, then it belongs to one of the 13 varieties $L_{1}, \ldots, L_{7}, L_{1}^{d}, \ldots, L_{6}^{d}$, which are defined by the following identities:

$$
\begin{aligned}
& L_{1}:(x y) z \approx x^{2}, x(y z) \approx x^{2} ; \\
& L_{2}:(x y) z \approx x^{2}, x(y z) \approx x y ; \\
& L_{3}:(x y) z \approx x y, x(y z) \approx x^{2} ; \\
& L_{4}:(x y) z \approx x z, x(y z) \approx x^{2} ; \\
& L_{5}:(x y) z \approx x y, x(y z) \approx x y ; \\
& L_{6}:(x y) z \approx x z, x(y z) \approx x y ; \\
& L_{7}:(x y) z \approx x z, x(y z) \approx x z .
\end{aligned}
$$

Proof We can derive the following three identities from $(x y) z \approx t_{1}$ and $x(y z) \approx t_{2}$ :

$$
\begin{aligned}
& t_{1}(x, y, z u) \approx(x y)(z u) \approx t_{2}(x y, z, u) ; \\
& t_{1}(x, y z, u) \approx(x(y z)) u \approx t_{2}(x, y, z) \cdot u ; \\
& x \cdot t_{1}(y, z, u) \approx x((y z) u) \approx t_{2}(x, y z, u) .
\end{aligned}
$$

In all the 16 cases marked by ' - ' in Table 2 , at least one of the above three identities contradicts the essentiality of the operation $f$. We work out the details only for the case $t_{1} \approx y^{2}, t_{2} \approx x y$ (here we will need the identity (3.1c)); the other cases are similar or even simpler (see Table 3): 


$$
\begin{aligned}
x z^{2} & \approx x \cdot t_{1}(y, z, u) \approx x((y z) u) \\
& \approx t_{2}(x, y z, u) \approx x(y z) \approx x y .
\end{aligned}
$$

Now it only remains to verify the entries marked by $L_{1} \cap L_{1}^{d}$ in Table 2 . These can be handled with the help of the identities (3.1); again, we provide details only for one case, namely for $t_{1} \approx y^{2}, t_{2} \approx x^{2}$, and refer to Table 3 for the remaining cases. Note that the variety $L_{1} \cap L_{1}^{d}$ is axiomatized by the identities $(x y) z \approx x(y z) \approx x^{2} \approx z^{2}$. This means that a groupoid $\mathbb{A}$ belongs to $L_{1} \cap L_{1}^{d}$ if and only if $\mathbb{A}$ is a semigroup and there is a constant $c \in A$ such that $x y z \approx x^{2} \approx c$. It is clear that such semigroups satisfy $(x y) z \approx y^{2}$ and $x(y z) \approx x^{2}$. Conversely, assume now that $(x y) z \approx y^{2}$ and $x(y z) \approx x^{2}$ hold in a groupoid A. Let us write out (3.1b):

$$
(y z)^{2} \approx t_{1}(x, y z, u) \approx(x(y z)) u \approx t_{2}(x, y, z) \cdot u \approx x^{2} u .
$$

We can conclude that $(y z)^{2}$ depends neither on $y$ nor on $z$, hence there is a constant $c \in A$ such that $(y z)^{2} \approx c$. Now let us use (3.1a):

$$
z^{2} \approx t_{1}(y, z, v u) \approx(y z)(v u) \approx t_{2}(y z, v, u) \approx(y z)^{2} .
$$

This implies that $z^{2}$ is constant $c$, hence $\mathbb{A}$ satisfies $(x y) z \approx x(y z) \approx x^{2} \approx c$; therefore, A $\in L_{1} \cap L_{1}^{d}$.

The entry marked by $L_{1} \cap L_{1}^{d}(!)$ in Table 2 is special in the sense that the identities $(x y) z \approx z^{2}, x(y z) \approx x^{2}$ do not guarantee laziness (see the example in Remark 2.3). Here (3.1a) yields $(z u)^{2} \approx(x y)^{2}$, i.e., $(x y)^{2}$ is constant. Since the only constant in $\mathcal{J}_{A} \cup \downarrow f$ is the diagonal operation $f(x)=x^{2}$, laziness implies that $x^{2}$ must be constant. Then we have $(x y) z \approx x(y z) \approx x^{2} \approx z^{2}$, hence $\mathbb{A} \in L_{1} \cap L_{1}^{d}$.

In order to complete the description of lazy groupoids, we still need to verify that every groupoid in the varieties $L_{1}, \ldots, L_{7}, L_{1}^{d}, \ldots, L_{6}^{d}$ defined in Lemma 3.3 is indeed lazy. In the following, whenever we use one of the two defining identities for any one of our varieties, we write " $\approx$ " or “2." to indicate whether we have used the first or the second identity (as listed in Lemma 3.3).

Lemma 3.4 If a groupoid $\mathbb{A}$ belongs to one of the 13 varieties $L_{1}, \ldots, L_{7}, L_{1}^{d}, \ldots, L_{6}^{d}$, then $\mathbb{A}$ is lazy.

Proof Assume that $\mathbb{A}$ is a groupoid in $L_{4}$ (the proof for the other varieties is very similar; see Table 4). We prove by term induction that every term of $\mathbb{A}$ is equivalent to $x$ or $x y$ (allowing that $x$ and $y$ are the same variable). Let $t$ be a term that contains at least two multiplications (i.e., at least three, not necessarily distinct variables). Then $t=s_{1} \cdot s_{2}$, where the terms $s_{1}$ and $s_{2}$ are shorter than $t$, hence, by the induction hypothesis, they are equivalent to a variable or to a product of two variables. Therefore, we have the following three possibilities with some (not necessarily distinct) variables $x, y, z, u$ : 


$$
\begin{aligned}
& s_{1} \approx x y, s_{2} \approx z \Longrightarrow s_{1} s_{2} \approx(x y) z \underset{1}{\approx} x z ; \\
& s_{1} \approx x, s_{2} \approx y z \Longrightarrow s_{1} s_{2} \approx x(y z) \approx x^{2} ; \\
& s_{1} \approx x y, s_{2} \approx z u \Longrightarrow s_{1} s_{2} \approx(x y)(z u) \stackrel{1}{\approx} x(z u) \stackrel{2}{\approx} x^{2} .
\end{aligned}
$$

Thus, every term over $L_{4}$ is indeed equivalent to a variable or a product of two variables, showing that every member of $L_{4}$ is a lazy groupoid.

Theorem 3.5 A groupoid $\mathbb{A}$ is lazy if and only if it belongs to one of the 15 varieties $L_{1}, \ldots, L_{7}, L_{1}^{d}, \ldots, L_{6}^{d}, \widetilde{U}, \widetilde{U}^{d}$.

Proof For essentially binary groupoids the "only if" part is covered by Lemma 3.3, while the "if" part follows from Lemma 3.4 and its dual. For essentially unary groupoids we can use Lemma 3.1. We do not need to list $U$ and $U^{d}$, since $U \subseteq L_{i}$ for every $i$ (cf. Fig. 1).

\section{Varieties of lazy groupoids}

It is easy to verify that the proper subvarieties of $U, \widetilde{U}$ and their duals are the varieties $L Z$ (left zero semigroups), $R Z$ (right zero semigroups), $Z$ (zero semigroups) and $T$ (trivial semigroups), as shown in Fig. 1:

$$
L Z: x \approx x y ; \quad R Z: x \approx y x ; \quad Z: x y \approx z u ; \quad T: x \approx y .
$$

Having determined all varieties of essentially at most unary lazy groupoids, let us now deal with subvarieties of $L_{1}, \ldots, L_{7}$. We get such subvarieties by adding some extra identities to the defining two identities of $L_{i}(i=1, \ldots, 7)$. Let $V$ be the intersection of $L_{i}$ and the variety defined by the identity $p \approx q$ (we assume $p \neq q$ ). Laziness of $L_{i}$ implies that $p$ and $q$ are both equivalent to a product of two (not necessarily distinct) variables over $V$. If one of $p$ and $q$ involves two different variables, say $p=x y$, but $q$ does not involve both $x$ and $y$, then $p \approx q$ implies that $f$ is essentially at most unary, hence $V$ is a subvariety of $U, U^{d}, \widetilde{U}$ or $\widetilde{U}^{d}$. If $x$ and $y$ both occur in $q$, then we get $x y \approx y x$ (commutativity). The remaining cases, when at most one variable occurs on both sides, are the following: $x^{2} \approx y^{2}$ (the main diagonal of the multiplication table is constant), $x^{2} \approx y$ (satisfied only by trivial groupoids), $x^{2} \approx x$ (idempotency), $x \approx y$ (satisfied only by trivial groupoids). Thus, in order to determine all non-unary subvarieties of $L_{i}$, we need to compute the intersection of $L_{i}$ by one or more of the following three varieties:

$$
I: x^{2} \approx x ; \quad D: x^{2} \approx y^{2} ; \quad C: x y \approx y x .
$$

Lemma 4.1 If a groupoid A belongs to one of the 21 varieties $L_{i} \cap V(i=1, \ldots, 7, V=I, D, C)$, then $\mathbb{A}$ is essentially at most unary, with the 
exception of the 3 varieties $L_{1} \cap D, L_{1} \cap C$ and $L_{7} \cap I=R B$ (here, and in the sequel, $R B$ denotes the variety of rectangular bands).

Proof We consider only $L_{3}$; the other cases can be seen in Table 5. Recall that $L_{3}$ is defined by $(x y) z \approx x y$ and $x(y z) \approx x^{2}$. As before, we will use $\approx$. one of these two identities, and we write $\approx, \approx$ and $\approx$ to indicate that we use the defining identity of $I, D$ and $C$, respectively. In the variety $L_{3} \cap I$ we have $x y \stackrel{I}{\approx}(x x) y \stackrel{1}{\approx} x x$, showing that $x y$ does not depend on $y$. In $L_{3} \cap D$ we have $x y \stackrel{1}{\approx}(x y)(z u) \stackrel{2}{\approx}(x y)^{2} \stackrel{D}{\approx} x^{2}$, which means again that $x y$ does not depend on $y$. Finally, in $L_{3} \cap C$ we can deduce $x y \stackrel{1 .}{\approx}(x y) z \stackrel{C}{\approx} z(x y) \stackrel{2}{\approx} z^{2}$, thus $x y$ depends neither on $x$ nor on $y$.

Theorem 4.2 There are 24 varieties of lazy groupoids, and they form the meet semilattice shown in Fig. 1 (semigroup varieties are indicated by filled circles).

Proof By Theorem 3.5 every lazy groupoid belongs to $L_{1}, \ldots, L_{7}, U, \widetilde{U}$ or to the duals of these varieties. We have already determined the subvarieties of $U$ and $\widetilde{U}$, and Lemma 4.1 describes all subvarieties of $L_{2}, \ldots, L_{7}$ (and their duals). Lemma 4.1 also implies that for $L_{1}$ and its dual we need to consider $L_{1} \cap D, L_{1} \cap C, L_{1}^{d} \cap D$, $L_{1}^{d} \cap C$ and any intersections of these. It is clear that $L_{1} \cap C$ is selfdual, i.e., $L_{1} \cap C=L_{1}^{d} \cap C$. The variety $L_{1} \cap D$ is defined by $(x y) z \approx x(y z) \approx x^{2} \approx z^{2}$, hence it it also selfdual: $L_{1} \cap D=L_{1}^{d} \cap D$. Therefore, the only variety that could be possibly missing from Fig. 1 is $L_{1} \cap D \cap C$. However, this coincides with $L_{1} \cap C$, as $L_{1} \cap C \subseteq L_{1} \cap D$. Indeed, we can derive $x^{2} \approx y^{2}$ from commutativity and the two defining identities of $L_{1}$ :

$$
x^{2} \stackrel{1}{\approx}(x z) y \stackrel{C}{\approx} y(x z) \stackrel{2}{\approx} y^{2}
$$

To prove that the 24 varieties in Fig. 1 are all distinct, we give the operation tables of their two-generated free algebras in "Appendix 2" (we include only one member of each pair of dual varieties, and we omit $T$ ). It remains to prove the containments indicated in Fig. 1. All of these are straightforward to verify, with the exception of $L_{1} \cap C \subseteq L_{1} \cap D$, which we have already proved.

\section{Characterizing lazy groupoids up to isomorphism}

We will give a concrete description of groupoids in the varieties $L_{1}, \ldots, L_{7}$ in this section (we do not write out the details for the dual varieties $L_{1}^{d}, \ldots, L_{6}^{d}$, and we also ignore the trivial unary cases $\widetilde{U}$ and $\widetilde{U}^{d}$ ).

Let us start with the variety $L_{7}$, which is the semigroup variety defined by $x y z \approx x z$. If $\mathbb{A} \in L_{7}$, then the set $E$ of the idempotent elements in $\mathbb{A}$ forms a rectangular band. We will prove below in Theorem 5.1 that $\mathbb{A}$ is an inflation of the rectangular band $\mathbb{E}=(E ; \cdot)$. This means that each idempotent $e \in E$ has a 
"neighborhood" $N_{e}$ containing $e$ such that $\left\{N_{e}: e \in E\right\}$ is a partition of $A$ (in particular, the only idempotent in $N_{e}$ is $e$ ), and for each $a \in N_{e}, b \in N_{f}$ we have $a b=e f$. In other words, $\mathbb{A}$ can be constructed from the rectangular band $\mathbb{E}$ by "inflating" each element $e \in E$ to a set $N_{e}$. Together with the well-known characterization of rectangular bands as direct products of a left zero semigroup and a right zero semigroup, this provides an explicit description of the members of $L_{7}$. This result appeared in [1] (and perhaps it has been known even earlier), but we include the proof for completeness.

Theorem 5.1 A groupoid $\mathbb{A}$ belongs to the variety $L_{7}$ if and only if there is a subset $E \subseteq A$ and a partition $\left\{N_{e}: e \in E\right\}$, such that $\mathbb{E}=(E ; \cdot)$ is a rectangular band, and

$$
\text { for all } e, f \in E \text { and } a \in N_{e}, b \in N_{f} \text {, we have } a^{2}=e \text { and } a b=e f \text {. }
$$

Proof Suppose that $\mathbb{A} \in L_{7}$, let $E=\left\{e \in A: e^{2}=e\right\}$ and let $N_{e}=\left\{a \in A: a^{2}=e\right\}$. Clearly, the sets $N_{e}(e \in E)$ are pairwise disjoint and nonempty (since $e \in N_{e}$ ); moreover, for all $a \in A$ we have $\left(a^{2}\right)^{2}=(a a)(a a) \stackrel{1}{=} a(a a) \stackrel{2}{=} a^{2}$, hence $e:=a^{2}$ is idempotent and $a \in N_{e}$. This shows that $\left\{N_{e}: e \in E\right\}$ is indeed a partition of $A$. The set $E$ is closed under multiplication, as $(e f)^{2}=(e f)(e f) \stackrel{1 .}{=} e(e f) \stackrel{2 .}{=} e f$ for all $e, f \in E$. Therefore $(E ; \cdot)$ is a subsemigroup, and it satisfies the identities $x y z \approx x z$ and $x^{2} \approx x$, hence it is a rectangular band. It only remains to prove $a b=e f$ for all $a \in N_{e}, b \in N_{f}$ :

$$
a b \stackrel{1 .}{=}(a a) b \stackrel{2 .}{=}(a a)(b b)=a^{2} b^{2}=e f .
$$

Now assume that $E \subseteq A$ such that $\mathbb{E}=(E ; \cdot)$ is a rectangular band, and $\left\{N_{e}: e \in E\right\}$ is a partition of $A$ such that (5.1) holds. In order to verify that $\mathbb{A}$ satisfies the identity $(x y) z \approx x z$, let us consider arbitrary elements $a, b, c \in A$. Then $a \in N_{e}, b \in N_{f}$ and $c \in N_{g}$ for some $e, f, g \in E$. From the second equality of (5.1) it follows that $a b=e f$ and $a c=e g$. Since $E$ is closed under multiplication, $h:=e f$ belongs to $E$, and $h^{2}=h$, as $\mathbb{E}$ is a band. The first equality of (5.1) implies that $h \in N_{h}$, and then we have $h c=h g$ by the second equality of (5.1). Putting everything together, we obtain $(a b) c=a c$ :

$$
(a b) c=(e f) c=h c=h g=(e f) g \stackrel{R B}{=} e g=a c,
$$

where in the equality marked by $R B$ we used the assumption that $\mathbb{E}$ is a rectangular band. We have proved that $\mathbb{A}$ satisfies the identity $(x y) z \approx x z$, and the identity $x(y z) \approx x z$ can be verified in a similar way, proving that $\mathbb{A} \in L_{7}$.

For the varieties $L_{1}, \ldots, L_{6}$, we will give similar characterizations in the following six theorems. Groupoids belonging to $L_{6}$ were described in [3] as unions of constant semigroups, which is essentially the same as Theorem 5.9 below, but our proof is different. Semigroups satisfying $x y z \approx x y$ were investigated and characterized in [2]; our Theorem 5.8 gives a different (and perhaps simpler) description. 
The characterizations will be given in terms of a partition $\left\{N_{e}: e \in E\right\}$ similarly to Theorem 5.1, but we will also need to specify a subset $S_{e} \subseteq N_{e}$ for all $e \in E$. Let us fix the notation for later reference:

Notation 5.2 Let $E \subseteq A$, and let $\left\{N_{e}: e \in E\right\}$ be a partition of $A$; moreover, for every $e \in E$, let $S_{e} \subseteq N_{e}$ such that $e \in S_{e}$.

Remark 5.3 Most of the time (with the exception of $L_{5}$ ), the setup will be the same as in Theorem 5.1: $E$ will be the set of idempotents, $N_{e}=\left\{a \in A: a^{2}=e\right\}$ and $S_{e}$ will be the set of factorizable elements in $N_{e}$, i.e., $S_{e}=N_{e} \cap\{a b: a, b \in A\}$. To see that $\left\{N_{e}: e \in E\right\}$ is indeed a partition of $A$, it suffices to note that $(x x)(x x) \approx x x$ holds in $L_{1}, \ldots, L_{6}$ (by the same argument as in the second sentence of the proof of Theorem 5.1). This implies that the square of any element is idempotent, therefore the sets $N_{e}$ cover $A$.

Theorem 5.4 A groupoid $\mathbb{A}$ belongs to the variety $L_{1}$ if and only if there is a partition $\left\{N_{e}: e \in E\right\}$ as in Notation 5.2, such that

$$
\begin{aligned}
& \text { for all } e, f \in E \text { and } a \in N_{e}, b \in N_{f} \text {, we have } a^{2}=e \text { and } \\
& a b=e, \quad \text { if } a \in S_{e}, \quad b \in S_{f} ; \\
& a b=e, \quad \text { if } a \in S_{e}, \quad b \in N_{f} \backslash S_{f} \text {; } \\
& a b=e, \quad \text { if } a \in N_{e} \backslash S_{e}, b \in S_{f} \text {; } \\
& a b \in S_{e} \text {, if } a \in N_{e} \backslash S_{e}, b \in N_{f} \backslash S_{f} \text {. }
\end{aligned}
$$

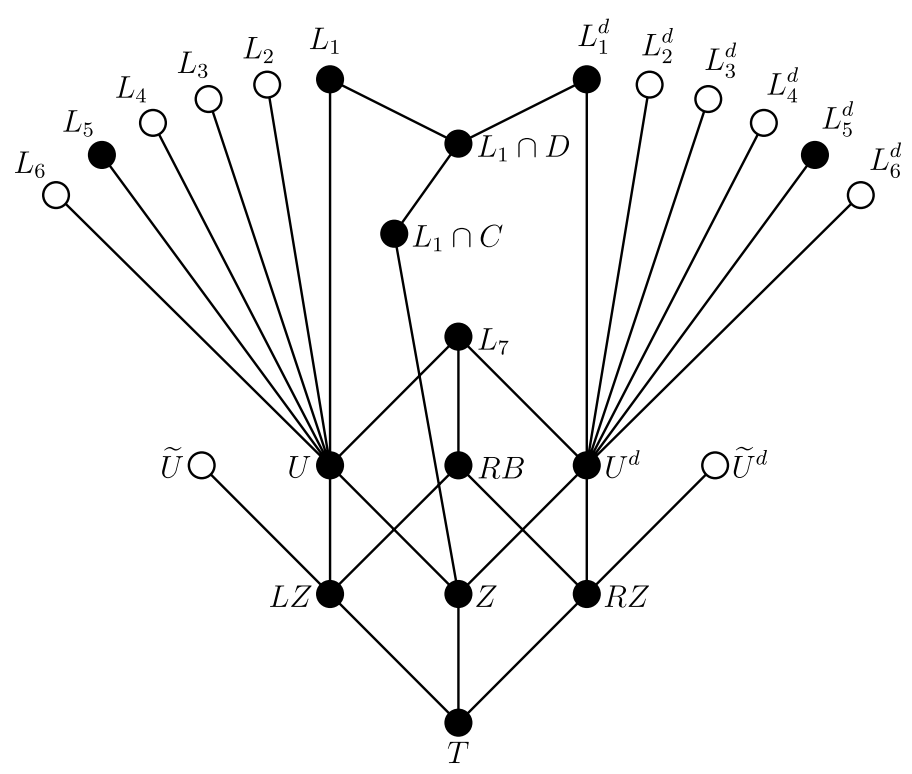

Fig. 1 The semilattice of lazy groupoid varieties 
Proof First assume that $\mathbb{A} \in L_{1}$ and recall that $L_{1}$ is defined by $(x y) z \approx x^{2}$ and $x(y z) \approx x^{2}$. Let us consider the partition described in Remark 5.3; then the first equality of (5.2) is automatically satisfied. For the other four statements, let $a \in N_{e}$ and $b \in N_{f}$. We have $(a b)^{2}=(a b)(a b) \stackrel{1 .}{=} a^{2}=e$, thus $a b \in N_{e}$. Therefore $a b \in S_{e}$, as $a b$ is obviously factorizable, and this verifies the last statement of (5.2). If $b \in S_{f}$, i.e., $b=b_{1} b_{2}$ for some $b_{1}, b_{2} \in A$, then $a b=a\left(b_{1} b_{2}\right) \stackrel{2 .}{=} a^{2}=e$. Similarly, if $a=a_{1} a_{2} \in S_{e}$, then $a b=\left(a_{1} a_{2}\right) b \stackrel{1 .}{=} a_{1}^{2} \stackrel{1}{=}\left(a_{1} a_{2}\right)^{2}=a^{2}=e$. This proves that (5.2) is satisfied.

Conversely, suppose that $\left\{N_{e}: e \in E\right\}$ is a partition of $A$ as in Notation 5.2, such that (5.2) holds. Let us compute $(a b) c$ and $a(b c)$ for $a \in N_{e}, b \in N_{f}, c \in N_{g}$. From (5.2) we see that $a b \in S_{e}$ and $b c \in S_{f}$, and then, again from (5.2), we obtain

$$
(a b) c=e=a^{2}, \quad a(b c)=e=a^{2} .
$$

This shows that $\mathbb{A}$ satisfies $(x y) z \approx x(y z) \approx x^{2}$, hence $\mathbb{A} \in L_{1}$.

Theorem 5.4 allows us to construct the multiplication table of any groupoid in $L_{1}$ as follows. Fix an arbitrary nonempty set $A$, and choose a partition $\left\{N_{e}: e \in E\right\}$ of $A$ as in Notation 5.2. Then define a multiplication on $A$ such that (5.2) is satisfied (if $a \in N_{e} \backslash S_{e}$ and $b \in N_{f} \backslash S_{f}$, then we can choose $a b$ to be any element of $S_{e}$ ). This gives a groupoid in $L_{1}$, and every member of $L_{1}$ can be obtained this way. A part of such an operation table can be seen in "Appendix 3". Only two blocks of the partition are displayed; the elements of $S_{e}$ are denoted by $e=s_{0}, s_{1}, \ldots$ and the elements of $N_{e} \backslash S_{e}$ are denoted by $a_{1}, a_{2}, \ldots$ (and similarly for $S_{f}$ and $N_{f} \backslash S_{f}$ ). However, this is only for notational convenience: these sets can have arbitrary cardinalities (not necessarily countable). "Appendix 3" contains similar tables for the varieties $L_{2}, \ldots, L_{6}$, illustrating the following five theorems.

Theorem 5.5 A groupoid $A$ belongs to the variety $L_{2}$ if and only if there is a partition $\left\{N_{e}: e \in E\right\}$ as in Notation 5.2, such that

$$
\begin{aligned}
& \text { for all } e, f \in E \text { and } a \in N_{e}, b \in N_{f}, \text { we have } a^{2}=e \text { and } \\
& \qquad \begin{array}{l}
a b=a f=e, \quad \text { if } a \in S_{e}, \quad b \in S_{f} ; \\
a b=a f=e, \quad \text { if } a \in S_{e}, \quad b \in N_{f} \backslash S_{f} ; \\
a b=a f \in S_{e}, \text { if } a \in N_{e} \backslash S_{e}, b \in S_{f} ; \\
a b=a f \in S_{e}, \text { if } a \in N_{e} \backslash S_{e}, b \in N_{f} \backslash S_{f} .
\end{array}
\end{aligned}
$$

Proof First assume that $\mathbb{A} \in L_{2}$ and recall that $L_{2}$ is defined by $(x y) z \approx x^{2}$ and $x(y z) \approx x y$. Let us consider the partition described in Remark 5.3. Let $a \in N_{e}$ and $b \in N_{f}$. We have $(a b)^{2}=(a b)(a b) \stackrel{1 .}{=} a^{2}=e$, hence $a b \in S_{e}$. Moreover, $a b \stackrel{2 .}{=} a(b b)=a f$, and if $a=a_{1} a_{2} \in S_{e}$, then $a b=\left(a_{1} a_{2}\right) b \stackrel{1 .}{=} a_{1}^{2} \stackrel{1 .}{=}\left(a_{1} a_{2}\right)^{2}=a^{2}=e$. This proves that (5.3) is satisfied. 
Conversely, suppose that $\left\{N_{e}: e \in E\right\}$ is a partition of $A$ as in Notation 5.2, such that (5.3) holds. Let us compute $(a b) c$ and $a(b c)$ for $a \in N_{e}, b \in N_{f}, c \in N_{g}$. From (5.3) we see that $a b \in S_{e}$ and $b c \in S_{f}$, and then, again from (5.3), we obtain

$$
(a b) c=e=a^{2}, \quad a(b c)=a f=a b .
$$

This shows that $\mathbb{A}$ satisfies $(x y) z \approx x^{2}$ and $x(y z) \approx x y$, hence $\mathbb{A} \in L_{2}$.

Theorem 5.6 A groupoid $\mathbb{A}$ belongs to the variety $L_{3}$ if and only if there is a partition $\left\{N_{e}: e \in E\right\}$ as in Notation 5.2, such that

$$
\begin{aligned}
& \text { for all } e, f \in E \text { and } a \in N_{e}, b \in N_{f}, \text { we have } a^{2}=e \text { and } \\
& \qquad \begin{array}{l}
a b=e, \text { if } a \in S_{e}, \quad b \in S_{f} ; \\
a b=e, \text { if } a \in S_{e}, \quad b \in N_{f} \backslash S_{f} ; \\
a b=e, \text { if } a \in N_{e} \backslash S_{e}, b \in S_{f} ; \\
a b \in E, \text { if } a \in N_{e} \backslash S_{e}, b \in N_{f} \backslash S_{f} .
\end{array}
\end{aligned}
$$

Proof First assume that $\mathbb{A} \in L_{3}$ and recall that $L_{3}$ is defined by $(x y) z \approx x y$ and $x(y z) \approx x^{2}$. Let us consider the partition described in Remark 5.3. Let $a \in N_{e}$ and $b \in N_{f}$; then $(a b)(a b) \stackrel{1 .}{=} a b$, which means that every product is idempotent (this already proves the last statement of (5.4)). In particular, if $a=a_{1} a_{2} \in S_{e}$, then $a^{2}=a$. However, the construction of the partition implies that $a^{2}=e$, thus we can conclude that $a=e$. (Note that this means that $S_{e}=\{e\}$.) Now we can write $a b$ as $a b=\left(a_{1} a_{2}\right) b \stackrel{1 .}{=} a_{1} a_{2}=a=e$. If $b=b_{1} b_{2} \in S_{f}$, then $a b=a\left(b_{1} b_{2}\right) \stackrel{2 .}{=} a^{2}=e$, hence (5.4) holds.

Conversely, suppose that $\left\{N_{e}: e \in E\right\}$ is a partition of $A$ as in Notation 5.2, such that (5.4) is satisfied. Let us compute $(a b) c$ and $a(b c)$ for $a \in N_{e}, b \in N_{f}, c \in N_{g}$. From (5.4) we see that $a b=: e^{\prime}$ and $b c=: f^{\prime}$ belong to $E$, and the construction of the partition implies that $e^{\prime} \in S_{e^{\prime}}$ and $f^{\prime} \in S_{f^{\prime}}$ (cf. Notation 5.2). Now we can calculate using (5.4) as follows:

$$
(a b) c=e^{\prime} c=e^{\prime}=a b, \quad a(b c)=a f^{\prime}=e=a^{2} .
$$

This proves that $\mathbb{A}$ satisfies $(x y) z \approx x y$ and $x(y z) \approx x^{2}$, hence $\mathbb{A} \in L_{3}$.

In the first half of the proof we observed that if the partition is chosen as in Remark 5.3, then each $S_{e}$ is a singleton. However, if we choose an arbitrary partition as in Notation 5.2 and we define the multiplication according to (5.4), then the resulting groupoid will be in $L_{3}$. This is not a contradiction: given such an operation table (like the one in "Appendix 3"), we can redefine the set $S_{e}$ so that $S_{e}=\{e\}$ (i.e., we "move" all elements of $S_{e}$ to $N_{e} \backslash S_{e}$ except for the element $e$ ) for each $e \in E$, without changing the multiplication table. Then the groupoid will still satisfy (5.4) for these new sets. 
Theorem 5.7 A groupoid $\mathbb{A}$ belongs to the variety $L_{4}$ if and only if there is a partition $\left\{N_{e}: e \in E\right\}$ as in Notation 5.2, such that

$$
\begin{aligned}
& \text { for all } e, f \in E \text { and } a \in N_{e}, b \in N_{f}, \text { we have } a^{2}=e \text { and } \\
& \qquad \begin{array}{l}
a b=e b=e, \quad \text { if } a \in S_{e}, \quad b \in S_{f} ; \\
a b=e b \in S_{e}, \text { if } a \in S_{e}, \quad b \in N_{f} \backslash S_{f} ; \\
a b=e b=e, \quad \text { if } a \in N_{e} \backslash S_{e}, b \in S_{f} ; \\
a b=e b \in S_{e}, \text { if } a \in N_{e} \backslash S_{e}, b \in N_{f} \backslash S_{f} .
\end{array}
\end{aligned}
$$

Proof First assume that $\mathbb{A} \in L_{4}$ and recall that $L_{4}$ is defined by $(x y) z \approx x z$ and $x(y z) \approx x^{2}$. Let us consider the partition described in Remark 5.3. If $a \in N_{e}$ and $b \in N_{f}$, then $(a b)^{2}=(a b)(a b) \stackrel{.}{=} a(a b) \stackrel{2}{=} a^{2}=e$, which means that $a b \in S_{e_{2}}$. We also have $a b \stackrel{=}{=}(a a) b=e b$; furthermore, if $b=b_{1} b_{2} \in S_{f}$, then $e b=e\left(b_{1} b_{2}\right) \stackrel{2 .}{=} e^{2}=e$. This proves that (5.5) holds.

Conversely, suppose that $\left\{N_{e}: e \in E\right\}$ is a partition of $A$ as in Notation 5.2, such that (5.5) is satisfied. Let us compute $(a b) c$ and $a(b c)$ for $a \in N_{e}, b \in N_{f}, c \in N_{g}$. From (5.5) we see that $a b \in S_{e}$ and $b c \in S_{f}$. Therefore, using (5.5) again, we obtain

$$
(a b) c=e c=a c, \quad a(b c)=e=a^{2} .
$$

This shows that $\mathbb{A}$ satisfies $(x y) z \approx x z$ and $x(y z) \approx x^{2}$, hence $\mathbb{A} \in L_{4}$.

Theorem 5.8 A groupoid $\mathbb{A}$ belongs to the variety $L_{5}$ if and only if there is a partition $\left\{N_{e}: e \in E\right\}$ as in Notation 5.2, such that

$$
\begin{aligned}
& \text { for all } e, f \in E \text { and } a \in N_{e}, b \in N_{f} \text {, we have } \\
& \qquad \begin{array}{l}
a b=a f=a, \quad \text { if } a \in S_{e}, \quad b \in S_{f} ; \\
a b=a f=a, \quad \text { if } a \in S_{e}, \quad b \in N_{f} \backslash S_{f} ; \\
a b=a f \in S_{e}, \text { if } a \in N_{e} \backslash S_{e}, b \in S_{f} ; \\
a b=a f \in S_{e}, \text { if } a \in N_{e} \backslash S_{e}, b \in N_{f} \backslash S_{f} .
\end{array}
\end{aligned}
$$

Proof First assume that $\mathbb{A} \in L_{5}$ and recall that $L_{5}$ is defined by $(x y) z \approx x y$ and $x(y z) \approx x y$. This time our partition will be different from that of Remark 5.3. We introduce an equivalence relation on $A$ : let us write $a \sim b$ if the right multiplications by $a$ and $b$ coincide, i.e., if $c a=c b$ for all $c \in A$ (in other words, the columns of $a$ and $b$ in the multiplication table are the same). Since $c a \stackrel{2 .}{=} c(a a)$ for every $c \in A$, we have $a \sim a^{2}$. As noted in Remark 5.3, $a^{2}$ is idempotent: $(a a)(a a) \stackrel{1 .}{=} a a$. Thus every equivalence class with respect to $\sim$ contains at least one idempotent element. Therefore, we can choose a complete set of representatives $E$ consisting of idempotent elements. Let $N_{e}$ denote the equivalence class of $e \in E$, and let $S_{e}$ be the set of factorizable elements in $N_{e}$, as before.

If $a \in N_{e}$ and $b \in N_{f}$, then $a b=a f$, as $b \sim f$. Moreover, $a \sim e$ implies that for every $c \in A$, we have $c e=c a \stackrel{2 .}{=} c(a f)$, hence $e \sim a f$, which means that $a f \in S_{e}$. If $a=a_{1} a_{2} \in S_{e}$, then $a b=a f=\left(a_{1} a_{2}\right) f \stackrel{1 .}{=} a_{1} a_{2}=a$. This proves that (5.6) holds. 
Conversely, suppose that $\left\{N_{e}: e \in E\right\}$ is a partition of $A$ as in Notation 5.2, such that (5.6) is satisfied. Let us compute $(a b) c$ and $a(b c)$ for $a \in N_{e}, b \in N_{f}, c \in N_{g}$. From (5.6) we see that $a b \in S_{e}$ and $b c \in S_{f}$. Therefore, using (5.6) again, we obtain

$$
(a b) c=a b, \quad a(b c)=a f=a b .
$$

This shows that $\mathbb{A}$ satisfies $(x y) z \approx x(y z) \approx x y$, hence $\mathbb{A} \in L_{5}$.

Theorem 5.9 A groupoid $\mathbb{A}$ belongs to the variety $L_{6}$ if and only if there is a partition $\left\{N_{e}: e \in E\right\}$ as in Notation 5.2, such that

$$
\text { for all } e, f \in E \text { and } a \in N_{e}, b \in N_{f} \text {, we have } a b=e f \in S_{e} \text {. }
$$

Proof First assume that $\mathbb{A} \in L_{6}$ and recall that $L_{6}$ is defined by $(x y) z \approx x z$ and $x(y z) \approx x y$. We use again the partition described in Remark 5.3. If $a \in N_{e}$ and $b \in N_{f}$, then $(a b)^{2}=(a b)(a b) \stackrel{1}{=} a(a b) \stackrel{2 .}{=} a^{2}=e$, therefore $a b \in S_{e}$. On the other hand, we can express $a b$ as $a b \stackrel{\text {. }}{=}(a a) b \stackrel{2}{=}(a a)(b b)=e f$, which proves that (5.7) holds.

Conversely, suppose that $\left\{N_{e}: e \in E\right\}$ is a partition of $A$ as in Notation 5.2, such that (5.7) is satisfied. Let us compute $(a b) c$ and $a(b c)$ for $a \in N_{e}, b \in N_{f}, c \in N_{g}$. From (5.7) we see that $a b \in S_{e}$ and $b c \in S_{f}$. Therefore, using (5.7) again, we obtain

$$
(a b) c=e g=a c, \quad a(b c)=e f=a b .
$$

This shows that $\mathbb{A}$ satisfies $(x y) z \approx x z$ and $x(y z) \approx x y$, hence $\mathbb{A} \in L_{6}$.

Acknowledgements The authors would like to thank the anonymous referee for their careful reading of the manuscript and for their valuable comments. This research was partially supported by the Hungarian Research, Development and Innovation Office under Grants K115518 and K128042, and by Grants 20391-3/2018/FEKUSTRAT and TUDFO/47138-1/2019-ITM of the Ministry for Innovation and Technology, Hungary. Open Access. FundRef: University of Szeged Open Access Fund, Grant Number: 4759.

Funding Open access funding provided by University of Szeged.

Open Access This article is licensed under a Creative Commons Attribution 4.0 International License, which permits use, sharing, adaptation, distribution and reproduction in any medium or format, as long as you give appropriate credit to the original author(s) and the source, provide a link to the Creative Commons licence, and indicate if changes were made. The images or other third party material in this article are included in the article's Creative Commons licence, unless indicated otherwise in a credit line to the material. If material is not included in the article's Creative Commons licence and your intended use is not permitted by statutory regulation or exceeds the permitted use, you will need to obtain permission directly from the copyright holder. To view a copy of this licence, visit http://creativecommons.org/licenses/by/4.0/. 


\section{Appendix 1: Detailed case analyses for the proofs of some lemmas}

Table 1 Summary of the proof of Lemma 3.2

\begin{tabular}{c|c|c}
$t_{1}$ & & \\
\hline \hline$x$ & $x y \approx t_{1}(x y, z, u) \approx t_{1}(x, y, z) u \approx x u$ & $x u$ does not depend on $u$ \\
\hline$y$ & $z \approx t_{1}(x y, z, u) \approx t_{1}(x, y, z) u \approx y u$ & $y u$ does not depend on $y, u$ \\
\hline$z$ & $u \approx t_{1}(x y, z, u) \approx t_{1}(x, y, z) u \approx z u$ & $z u$ does not depend on $z$ \\
\hline$z y$ & $u z \approx t_{1}(x y, z, u) \approx t_{1}(x, y, z) u$ & \\
\hline$z x$ & $\begin{array}{c}u(x y) \approx t_{1}(x y, z, u) \approx t_{1}(x, y, z) u \\
\approx(z x) u \approx t_{1}(z, x, u) \approx u z\end{array}$ & $u z$ does not depend on $z$ \\
\hline$y x$ & $w v \approx t_{1}(v, w, x y) \approx(v w)(x y)$ & \\
& $\approx t_{1}(x y, v w, u) \approx t_{1}(x, y, v w) u$ & \\
& $\approx(y x) u \approx t_{1}(y, x, u) \approx x y$ & $x y$ does not depend on $x, y$
\end{tabular}

Table 2 The 36 cases of Lemma 3.3

\begin{tabular}{|c|c|c|c|c|c|c|}
\hline & $x^{2}$ & $y^{2}$ & $z^{2}$ & $x y$ & $y z$ & $x z$ \\
\hline$x^{2}$ & $L_{1}$ & $L_{1} \cap L_{1}^{d}$ & $L_{1} \cap L_{1}^{d}$ & $L_{2}$ & - & - \\
\hline$y^{2}$ & $L_{1} \cap L_{1}^{d}$ & $L_{1} \cap L_{1}^{d}$ & $L_{1} \cap L_{1}^{d}$ & - & - & - \\
\hline$z^{2}$ & $L_{1} \cap L_{1}^{d}(!)$ & $L_{1} \cap L_{1}^{d}$ & $L_{1}^{d}$ & - & $L_{3}^{d}$ & $L_{4}^{d}$ \\
\hline$x y$ & $L_{3}$ & - & - & $L_{5}$ & - & - \\
\hline$y z$ & - & - & $L_{2}^{d}$ & - & $L_{5}^{d}$ & $L_{6}^{d}$ \\
\hline$x z$ & $L_{4}$ & - & - & $L_{6}$ & - & $L_{7}=L_{7}^{d}$ \\
\hline
\end{tabular}


Table 3 Summary of the proof of Lemma 3.3

\begin{tabular}{|c|c|c|c|}
\hline$t_{1}$ & $t_{2}$ & & \\
\hline$x^{2}$ & $y z$ & (a) & $z u \approx t_{2}(x y, z, u) \approx t_{1}(x, y, z u) \approx x^{2}$ \\
\hline$y^{2}$ & $y z$ & (a) & $z u \approx t_{2}(x y, z, u) \approx t_{1}(x, y, z u) \approx y^{2}$ \\
\hline$x y$ & $y^{2}$ & (a) & $x y \approx t_{1}(x, y, z u) \approx t_{2}(x y, z, u) \approx z^{2}$ \\
\hline$x y$ & $z^{2}$ & (a) & $x y \approx t_{1}(x, y, z u) \approx t_{2}(x y, z, u) \approx u^{2}$ \\
\hline$x y$ & $y z$ & (a) & $x y \approx t_{1}(x, y, z u) \approx t_{2}(x y, z, u) \approx z u$ \\
\hline$y z$ & $x^{2}$ & (b) & $z u \approx t_{1}(y, z, u) \approx(y z) u \approx t_{1}(x, y z, u) \approx t_{2}(x, y, z) u \approx x^{2} u$ \\
\hline$y z$ & $y^{2}$ & (b) & $z u \approx t_{1}(y, z, u) \approx(y z) u \approx t_{1}(x, y z, u) \approx t_{2}(x, y, z) u \approx y^{2} u$ \\
\hline$y z$ & $x y$ & (b) & $z u \approx t_{1}(y, z, u) \approx(y z) u \approx t_{1}(x, y z, u) \approx t_{2}(x, y, z) u \approx(x y) u$ \\
\hline$x z$ & $y^{2}$ & (b) & $x u \approx t_{1}(x, y z, u) \approx t_{2}(x, y, z) u \approx y^{2} u$ \\
\hline$x z$ & $z^{2}$ & (b) & $x u \approx t_{1}(x, y z, u) \approx t_{2}(x, y, z) u \approx z^{2} u$ \\
\hline$x z$ & $y z$ & (b) & $x u \approx t_{1}(x, y z, u) \approx t_{2}(x, y, z) u \approx(y z) u$ \\
\hline$y^{2}$ & $x y$ & (c) & $x y \approx t_{2}(x, y, z) \approx x(y z) \approx t_{2}(x, y z, u) \approx x t_{1}(y, z, u) \approx x z^{2}$ \\
\hline$z^{2}$ & $x y$ & (c) & $x y \approx t_{2}(x, y, z) \approx x(y z) \approx t_{2}(x, y z, u) \approx x t_{1}(y, z, u) \approx x u^{2}$ \\
\hline$x^{2}$ & $x z$ & (c) & $x u \approx t_{2}(x, y z, u) \approx x t_{1}(y, z, u) \approx x y^{2}$ \\
\hline$y^{2}$ & $x z$ & (c) & $x u \approx t_{2}(x, y z, u) \approx x t_{1}(y, z, u) \approx x z^{2}$ \\
\hline$x y$ & $x z$ & (c) & $x u \approx t_{2}(x, y z, u) \approx x t_{1}(y, z, u) \approx x(y z)$ \\
\hline$x^{2}$ & $y^{2}$ & (a) & $x^{2} \approx t_{1}(x, y, z u) \approx t_{2}(x y, z, u) \approx z^{2}$ \\
\hline$x^{2}$ & $z^{2}$ & (a) & $x^{2} \approx t_{1}(x, y, z u) \approx t_{2}(x y, z, u) \approx u^{2}$ \\
\hline$y^{2}$ & $y^{2}$ & (a) & $y^{2} \approx t_{1}(x, y, z u) \approx t_{2}(x y, z, u) \approx z^{2}$ \\
\hline$y^{2}$ & $z^{2}$ & (a) & $y^{2} \approx t_{1}(x, y, z u) \approx t_{2}(x y, z, u) \approx u^{2}$ \\
\hline$y^{2}$ & $x^{2}$ & (a) & $z^{2} \approx t_{1}(y, z, v u) \approx t_{2}(y z, v, u) \approx(y z)^{2}$ \\
\hline & & (b) & $\approx t_{1}(x, y z, u) \approx t_{2}(x, y, z) u \approx x^{2} u$ \\
\hline$z^{2}$ & $y^{2}$ & $\begin{array}{l}\text { (a) } \\
\text { (c) }\end{array}$ & $\begin{array}{c}y^{2} \approx t_{2}(x u, y, z) \approx t_{1}(x, u, y z) \approx(y z)^{2} \\
\approx t_{2}(x, y z, u) \approx x t_{1}(y, z, u) \approx x u^{2}\end{array}$ \\
\hline
\end{tabular}


Table 4 Summary of the proof of Lemma 3.4

$$
\begin{aligned}
& L_{1}: \quad s_{1} \approx x y, \quad s_{2} \approx z \quad \Longrightarrow s_{1} s_{2} \approx(x y) z \approx x^{2} \\
& s_{1} \approx x, \quad s_{2} \approx y z \quad \Longrightarrow s_{1} s_{2} \approx x(y z) \stackrel{2}{\approx} x^{2} \\
& s_{1} \approx x y, s_{2} \approx z u \Longrightarrow s_{1} s_{2} \approx(x y)(z u) \stackrel{1}{\approx} x^{2} \\
& L_{2}: \quad s_{1} \approx x y, \quad s_{2} \approx z \quad \Longrightarrow s_{1} s_{2} \approx(x y) z \approx x^{2} \\
& s_{1} \approx x, \quad s_{2} \approx y z \quad \Longrightarrow s_{1} s_{2} \approx x(y z) \stackrel{2}{\approx} x y \\
& s_{1} \approx x y, s_{2} \approx z u \Longrightarrow s_{1} s_{2} \approx(x y)(z u) \stackrel{1}{\approx} x^{2} \\
& L_{3}: \quad s_{1} \approx x y, \quad s_{2} \approx z \quad \Longrightarrow s_{1} s_{2} \approx(x y) z \approx x y \\
& s_{1} \approx x, \quad s_{2} \approx y z \quad \Longrightarrow s_{1} s_{2} \approx x(y z) \stackrel{2}{\approx} x^{2} \\
& s_{1} \approx x y, s_{2} \approx z u \Longrightarrow s_{1} s_{2} \approx(x y)(z u) \stackrel{1}{\approx} x y \\
& L_{4}: \quad s_{1} \approx x y, \quad s_{2} \approx z \quad \Longrightarrow s_{1} s_{2} \approx(x y) z \stackrel{1}{\approx} x z \\
& s_{1} \approx x, \quad s_{2} \approx y z \quad \Longrightarrow s_{1} s_{2} \approx x(y z) \stackrel{2}{\approx} x^{2} \\
& s_{1} \approx x y, s_{2} \approx z u \Longrightarrow s_{1} s_{2} \approx(x y)(z u) \stackrel{1}{\approx} x(z u) \stackrel{2}{\approx} x^{2} \\
& L_{5}: \quad s_{1} \approx x y, s_{2} \approx z \quad \Longrightarrow s_{1} s_{2} \approx(x y) z \approx x y \\
& s_{1} \approx x, \quad s_{2} \approx y z \quad \Longrightarrow s_{1} s_{2} \approx x(y z) \stackrel{2}{\approx} x y \\
& s_{1} \approx x y, s_{2} \approx z u \Longrightarrow s_{1} s_{2} \approx(x y)(z u) \stackrel{1}{\approx} x y \\
& L_{6}: \quad s_{1} \approx x y, s_{2} \approx z \quad \Longrightarrow s_{1} s_{2} \approx(x y) z \stackrel{1}{\approx} x z \\
& s_{1} \approx x, \quad s_{2} \approx y z \quad \Longrightarrow s_{1} s_{2} \approx x(y z) \stackrel{2}{\approx} x y \\
& s_{1} \approx x y, s_{2} \approx z u \Longrightarrow s_{1} s_{2} \approx(x y)(z u) \stackrel{1}{\approx} x(z u) \stackrel{2}{\approx} x z \\
& L_{7}: \quad s_{1} \approx x y, \quad s_{2} \approx z \quad \Longrightarrow s_{1} s_{2} \approx(x y) z \stackrel{1}{\approx} x z \\
& s_{1} \approx x, \quad s_{2} \approx y z \quad \Longrightarrow s_{1} s_{2} \approx x(y z) \stackrel{2}{\approx} x z \\
& s_{1} \approx x y, s_{2} \approx z u \Longrightarrow s_{1} s_{2} \approx(x y)(z u) \stackrel{1}{\approx} x(z u) \stackrel{2}{\approx} x u
\end{aligned}
$$


Table 5 Summary of the proof of Lemma 4.1

\begin{tabular}{|c|c|c|}
\hline$L_{1} \cap I$ & $x y \stackrel{I}{\approx}(x x) y \stackrel{1}{\approx} x^{2}$ & $x y$ does not depend on $y$ \\
\hline$L_{2} \cap I$ & $x y \stackrel{I}{\approx}(x x) y \stackrel{1}{\approx} x^{2}$ & $x y$ does not depend on $y$ \\
\hline$L_{2} \cap D$ & $x y \stackrel{2}{\approx} x(y y) \stackrel{D}{\approx} x(x x)$ & $x y$ does not depend on $y$ \\
\hline$L_{2} \cap C$ & $x y \stackrel{2}{\approx} x(y z) \stackrel{C}{\approx}(y z) x \stackrel{1}{\approx} y^{2}$ & $x y$ does not depend on $x$ \\
\hline$L_{3} \cap I$ & $x y \stackrel{I}{\approx}(x x) y \stackrel{1}{\approx} x^{2}$ & $x y$ does not depend on $y$ \\
\hline$L_{3} \cap D$ & $x y \stackrel{1}{\approx}(x y)(z u) \stackrel{2}{\approx}(x y)^{2} \stackrel{D}{\approx} x^{2}$ & $x y$ does not depend on $y$ \\
\hline$L_{3} \cap C$ & $x y \stackrel{1}{\approx}(x y) z \stackrel{C}{\approx} z(x y) \stackrel{2}{\approx} z^{2}$ & $x y$ does not depend on $y$ \\
\hline$L_{4} \cap I$ & $x y \stackrel{I}{\approx} x(y y) \stackrel{2}{\approx} x^{2}$ & $x y$ does not depend on $y$ \\
\hline$L_{4} \cap D$ & $x y \stackrel{1}{\approx}(x x) y \stackrel{D}{\approx}(y y) y$ & $x y$ does not depend on $x$ \\
\hline$L_{4} \cap C$ & $x y \stackrel{1}{\approx}(x z) y \stackrel{C}{\approx} y(x z) \stackrel{2}{\approx} y^{2}$ & $x y$ does not depend on $x$ \\
\hline$L_{5} \cap I$ & $x y \stackrel{I}{\approx}(x x) y \stackrel{1}{\approx} x^{2}$ & $x y$ does not depend on $y$ \\
\hline$L_{5} \cap D$ & $x y \stackrel{2}{\approx} x(y y) \stackrel{D}{\approx} x(x x)$ & $x y$ does not depend on $y$ \\
\hline$L_{5} \cap C$ & $x y \stackrel{1}{\approx}(x y) z \stackrel{C}{\approx} z(x y) \stackrel{2}{\approx} z x$ & $x y$ does not depend on $y$ \\
\hline$L_{6} \cap I$ & $x y \stackrel{I}{\approx}(x y)(x y) \stackrel{1}{\approx} x(x y) \stackrel{2}{\approx} x^{2}$ & $x y$ does not depend on $y$ \\
\hline$L_{6} \cap D$ & $x y \stackrel{2}{\approx} x(y y) \stackrel{D}{\approx} x(x x)$ & $x y$ does not depend on $y$ \\
\hline$L_{6} \cap C$ & $x y \stackrel{2}{\approx} x(y z) \stackrel{C}{\approx}(z y) x \stackrel{1}{\approx} z x$ & $x y$ does not depend on $y$ \\
\hline$L_{7} \cap D$ & $x y \stackrel{2}{\approx} x(y y) \stackrel{D}{\approx} x(x x)$ & $x y$ does not depend on $y$ \\
\hline$L_{7} \cap C$ & $x y \stackrel{1}{\approx}(x z) y \stackrel{C}{\approx} y(x z) \stackrel{2}{\approx} y z$ & $x y$ does not depend on $x$ \\
\hline
\end{tabular}




\section{Appendix 2: Two-generated free algebras in lazy groupoid varieties}

\begin{tabular}{|c|c|c|c|c|c|c|c|c|c|c|c|c|c|}
\hline$L_{1}$ & $x$ & $y$ & $x^{2}$ & $y^{2}$ & $x y$ & $y x$ & $L_{2}$ & $x$ & $y$ & $x^{2}$ & $y^{2}$ & $x y$ & $y x$ \\
\hline$x$ & $x^{2}$ & $x y$ & $x^{2}$ & $x^{2}$ & $x^{2}$ & $x^{2}$ & $x$ & $x^{2}$ & $x y$ & $x^{2}$ & $x y$ & $x^{2}$ & $x y$ \\
\hline$y$ & $y x$ & $y^{2}$ & $y^{2}$ & $y^{2}$ & $y^{2}$ & $y^{2}$ & $y$ & $y x$ & $y^{2}$ & $y x$ & $y^{2}$ & $y x$ & $y^{2}$ \\
\hline$x^{2}$ & $x^{2}$ & $x^{2}$ & $x^{2}$ & $x^{2}$ & $x^{2}$ & $x^{2}$ & $x^{2}$ & $x^{2}$ & $x^{2}$ & $x^{2}$ & $x^{2}$ & $x^{2}$ & $x^{2}$ \\
\hline$y^{2}$ & $y^{2}$ & 2 & 2 & $y^{2}$ & $y^{2}$ & $y^{2}$ & $y^{2}$ & $y^{2}$ & $y^{2}$ & $y^{2}$ & $y^{2}$ & $y^{2}$ & $y^{2}$ \\
\hline$x y$ & $x^{2}$ & $x^{2}$ & $x^{2}$ & $x^{2}$ & $x^{2}$ & $x^{2}$ & $x y$ & $x^{2}$ & $x^{2}$ & $x^{2}$ & $x^{2}$ & $x^{2}$ & $x^{2}$ \\
\hline$y x$ & $y^{2}$ & $y^{2}$ & $y^{2}$ & $y^{2}$ & $y^{2}$ & $y^{2}$ & $y x$ & $y^{2}$ & $y^{2}$ & $y^{2}$ & $y^{2}$ & $y^{2}$ & $y^{2}$ \\
\hline
\end{tabular}

\begin{tabular}{c|ccccc}
$L_{1} \cap D$ & $x$ & $y$ & $x^{2}$ & $x y$ & $y x$ \\
\hline$x$ & $x^{2}$ & $x y$ & $x^{2}$ & $x^{2}$ & $x^{2}$ \\
$y$ & $y x$ & $x^{2}$ & $x^{2}$ & $x^{2}$ & $x^{2}$ \\
$x^{2}$ & $x^{2}$ & $x^{2}$ & $x^{2}$ & $x^{2}$ & $x^{2}$ \\
$x y$ & $x^{2}$ & $x^{2}$ & $x^{2}$ & $x^{2}$ & $x^{2}$ \\
$y x$ & $x^{2}$ & $x^{2}$ & $x^{2}$ & $x^{2}$ & $x^{2}$
\end{tabular}

\begin{tabular}{c|cccccc}
$L_{3}$ & $x$ & $y$ & $x^{2}$ & $y^{2}$ & $x y$ & $y x$ \\
\hline$x$ & $x^{2}$ & $x y$ & $x^{2}$ & $x^{2}$ & $x^{2}$ & $x^{2}$ \\
$y$ & $y x$ & $y^{2}$ & $y^{2}$ & $y^{2}$ & $y^{2}$ & $y^{2}$ \\
$x^{2}$ & $x^{2}$ & $x^{2}$ & $x^{2}$ & $x^{2}$ & $x^{2}$ & $x^{2}$ \\
$y^{2}$ & $y^{2}$ & $y^{2}$ & $y^{2}$ & $y^{2}$ & $y^{2}$ & $y^{2}$ \\
$x y$ & $x y$ & $x y$ & $x y$ & $x y$ & $x y$ & $x y$ \\
$y x$ & $y x$ & $y x$ & $y x$ & $y x$ & $y x$ & $y x$
\end{tabular}

\begin{tabular}{c|cccc}
$L_{1} \cap C$ & $x$ & $y$ & $x^{2}$ & $x y$ \\
\hline$x$ & $x^{2}$ & $x y$ & $x^{2}$ & $x^{2}$ \\
$y$ & $x y$ & $x^{2}$ & $x^{2}$ & $x^{2}$ \\
$x^{2}$ & $x^{2}$ & $x^{2}$ & $x^{2}$ & $x^{2}$ \\
$x y$ & $x^{2}$ & $x^{2}$ & $x^{2}$ & $x^{2}$
\end{tabular}

\begin{tabular}{c|cccccc}
$L_{4}$ & $x$ & $y$ & $x^{2}$ & $y^{2}$ & $x y$ & $y x$ \\
\hline$x$ & $x^{2}$ & $x y$ & $x^{2}$ & $x^{2}$ & $x^{2}$ & $x^{2}$ \\
$y$ & $y x$ & $y^{2}$ & $y^{2}$ & $y^{2}$ & $y^{2}$ & $y^{2}$ \\
$x^{2}$ & $x^{2}$ & $x y$ & $x^{2}$ & $x^{2}$ & $x^{2}$ & $x^{2}$ \\
$y^{2}$ & $y x$ & $y^{2}$ & $y^{2}$ & $y^{2}$ & $y^{2}$ & $y^{2}$ \\
$x y$ & $x^{2}$ & $x y$ & $x^{2}$ & $x^{2}$ & $x^{2}$ & $x^{2}$ \\
$y x$ & $y x$ & $y^{2}$ & $y^{2}$ & $y^{2}$ & $y^{2}$ & $y^{2}$
\end{tabular}




\begin{tabular}{c|cccccc}
$L_{5}$ & $x$ & $y$ & $x^{2}$ & $y^{2}$ & $x y$ & $y x$ \\
\hline$x$ & $x^{2}$ & $x y$ & $x^{2}$ & $x y$ & $x^{2}$ & $x y$ \\
$y$ & $y x$ & $y^{2}$ & $y x$ & $y^{2}$ & $y x$ & $y^{2}$ \\
$x^{2}$ & $x^{2}$ & $x^{2}$ & $x^{2}$ & $x^{2}$ & $x^{2}$ & $x^{2}$ \\
$y^{2}$ & $y^{2}$ & $y^{2}$ & $y^{2}$ & $y^{2}$ & $y^{2}$ & $y^{2}$ \\
$x y$ & $x y$ & $x y$ & $x y$ & $x y$ & $x y$ & $x y$ \\
$y x$ & $y x$ & $y x$ & $y x$ & $y x$ & $y x$ & $y x$
\end{tabular}

\begin{tabular}{c|cccc}
$U$ & $x$ & $y$ & $x^{2}$ & $y^{2}$ \\
\hline$x$ & $x^{2}$ & $x^{2}$ & $x^{2}$ & $x^{2}$ \\
$y$ & $y^{2}$ & $y^{2}$ & $y^{2}$ & $y^{2}$ \\
$x^{2}$ & $x^{2}$ & $x^{2}$ & $x^{2}$ & $x^{2}$ \\
$y^{2}$ & $y^{2}$ & $y^{2}$ & $y^{2}$ & $y^{2}$ \\
& & & &
\end{tabular}

\begin{tabular}{c|rrrrrr}
$L_{6}$ & $x$ & $y$ & $x^{2}$ & $y^{2}$ & $x y$ & $y x$ \\
\hline$x$ & $x^{2}$ & $x y$ & $x^{2}$ & $x y$ & $x^{2}$ & $x y$ \\
$y$ & $y x$ & $y^{2}$ & $y x$ & $y^{2}$ & $y x$ & $y^{2}$ \\
$x^{2}$ & $x^{2}$ & $x y$ & $x^{2}$ & $x y$ & $x^{2}$ & $x y$ \\
$y^{2}$ & $y x$ & $y^{2}$ & $y x$ & $y^{2}$ & $y x$ & $y^{2}$ \\
$x y$ & $x^{2}$ & $x y$ & $x^{2}$ & $x y$ & $x^{2}$ & $x y$ \\
$y x$ & $y x$ & $y^{2}$ & $y x$ & $y^{2}$ & $y x$ & $y^{2}$
\end{tabular}

\begin{tabular}{c|cccc}
$\widetilde{U}$ & $x$ & $y$ & $x^{2}$ & $y^{2}$ \\
\hline$x$ & $x^{2}$ & $x^{2}$ & $x^{2}$ & $x^{2}$ \\
$y$ & $y^{2}$ & $y^{2}$ & $y^{2}$ & $y^{2}$ \\
$x^{2}$ & $x$ & $x$ & $x$ & $x$ \\
$y^{2}$ & $y$ & $y$ & $y$ & $y$
\end{tabular}

\begin{tabular}{|c|c|c|c|c|c|c|c|c|c|c|}
\hline$L_{7}$ & $x$ & $y$ & $x^{2}$ & $y^{2}$ & $x y$ & $y x$ & $Z$ & $x$ & $y$ & $x y$ \\
\hline$x$ & $x^{2}$ & $x y$ & $x^{2}$ & $x y$ & $x y$ & $x^{2}$ & $x$ & $x y$ & $x y$ & $x y$ \\
\hline$y$ & $y x$ & $y^{2}$ & $y x$ & $y^{2}$ & $y^{2}$ & $y x$ & $y$ & $x y$ & $x y$ & $x y$ \\
\hline$x^{2}$ & $x^{2}$ & $x y$ & $x^{2}$ & $x y$ & $x y$ & $x^{2}$ & $x y$ & $x y$ & $x y$ & $x y$ \\
\hline$y^{2}$ & $y x$ & $y^{2}$ & $y x$ & $y^{2}$ & $y^{2}$ & $y x$ & & & & \\
\hline$x y$ & $x^{2}$ & $x y$ & $x^{2}$ & $x y$ & $x y$ & $x^{2}$ & & & & \\
\hline$y x$ & $y x$ & $y^{2}$ & $y x$ & $y^{2}$ & $y^{2}$ & $y x$ & & & & \\
\hline
\end{tabular}




\begin{tabular}{c|cccc}
$R B$ & $x$ & $y$ & $x y$ & $y x$ \\
\hline$x$ & $x$ & $x y$ & $x y$ & $x$ \\
$y$ & $y x$ & $y$ & $y$ & $y x$ \\
$x y$ & $x$ & $x y$ & $x y$ & $x$ \\
$y x$ & $y x$ & $y$ & $y$ & $y x$
\end{tabular}

\begin{tabular}{c|cc}
$L Z$ & $x$ & $y$ \\
\hline$x$ & $x$ & $x$ \\
$y$ & $y$ & $y$
\end{tabular}

\section{Appendix 3: Operation tables of lazy groupoids}

\begin{tabular}{|c|c|c|c|c|c|c|c|c|c|c|c|c|c|c|c|}
\hline$L_{1}$ & $e$ & $s_{1}$ & $s_{2}$ & $\ldots$ & $a_{1}$ & $a_{2}$ & $\ldots$ & $f$ & $t_{1}$ & $t_{2}$ & $\ldots$ & $b_{1}$ & $b_{2}$ & $\ldots$ & $\ldots$ \\
\hline$s_{0}=e$ & $e$ & $e$ & $e$ & $\ldots$ & $e$ & $e$ & $\ldots$ & $e$ & $e$ & $e$ & $\ldots$ & $e$ & $e$ & $\ldots$ & $\ldots$ \\
\hline$s_{1} \in S_{e}$ & $e$ & $e$ & $e$ & $\ldots$ & $e$ & $e$ & $\ldots$ & $e$ & $e$ & $e$ & $\ldots$ & $e$ & $e$ & $\ldots$ & $\ldots$ \\
\hline$s_{2} \in S_{e}$ & $e$ & $e$ & $e$ & $\ldots$ & $e$ & $e$ & $\ldots$ & $e$ & $e$ & $e$ & $\ldots$ & $e$ & $e$ & $\ldots$ & $\ldots$ \\
\hline : & : & : & : & & : & : & & : & : & 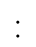 & & : & & & \\
\hline$a_{1} \notin S_{e}$ & $e$ & $e$ & $e$ & $\ldots$ & $e$ & $\in S_{e}$ & $\ldots$ & $e$ & $e$ & $e$ & $\ldots$ & $\in S_{e}$ & $\in S_{e}$ & $\ldots$ & $\ldots$ \\
\hline$a_{2} \notin S_{e}$ & $e$ & $e$ & $e$ & $\ldots$ & $\in S_{e}$ & $e$ & $\ldots$ & $e$ & $e$ & $e$ & $\ldots$ & $\in S_{e}$ & $\in S_{e}$ & $\ldots$ & $\ldots$ \\
\hline & $\vdots$ & $\vdots$ & $\vdots$ & & $\vdots$ & $\vdots$ & & $\vdots$ & : & : & & : & $\vdots$ & & \\
\hline$t_{0}=f$ & $f$ & $f$ & $f$ & $\ldots$ & $f$ & $f$ & $\ldots$ & $f$ & $f$ & $f$ & $\ldots$ & $f$ & $f$ & $\ldots$ & $\ldots$ \\
\hline$t_{1} \in S_{f}$ & $f$ & $f$ & $f$ & $\ldots$ & $f$ & $f$ & $\ldots$ & $f$ & $f$ & $f$ & $\ldots$ & $f$ & $f$ & $\ldots$ & $\ldots$ \\
\hline$t_{2} \in S_{f}$ & $f$ & $f$ & $f$ & $\ldots$ & $f$ & $f$ & $\ldots$ & $f$ & $f$ & $f$ & $\ldots$ & $f$ & $f$ & $\ldots$ & $\ldots$ \\
\hline : & $\vdots$ & : & : & & : & : & & $\vdots$ & : & : & & : & : & & \\
\hline$b_{1} \notin S_{f}$ & $f$ & $f$ & $f$ & $\ldots$ & $\in S_{f}$ & $\in S_{f}$ & $\ldots$ & $f$ & $f$ & $f$ & $\ldots$ & $f$ & $\in S_{f}$ & $\ldots$ & $\ldots$ \\
\hline$b_{2} \notin S_{f}$ & $f$ & $f$ & $f$ & $\ldots$ & $\in S_{f}$ & $\in S_{f}$ & $\ldots$ & $f$ & $f$ & $f$ & $\ldots$ & $\in S_{f}$ & $f$ & $\ldots$ & $\ldots$ \\
\hline : & : & : & : & & : & : & & $\therefore$ & : & : & & : & : & & \\
\hline$:$ & $:$ & $:$ & $:$ & & $:$ & $:$ & & $:$ & : & : & & : & $:$ & & \\
\hline & : & $:$ & $\vdots$ & & & . & & & : & : & & : & $\therefore$ & & \\
\hline
\end{tabular}




\begin{tabular}{|c|c|c|c|c|c|c|c|c|c|c|c|c|c|c|c|}
\hline$L_{2}$ & $e$ & $s_{1}$ & $s_{2}$ & $\ldots$ & $a_{1}$ & $a_{2}$ & $\ldots$ & $f$ & $t_{1}$ & $t_{2}$ & $\ldots$ & $b_{1}$ & $b_{2}$ & $\ldots$ & $\ldots$ \\
\hline$s_{0}=e$ & $e$ & $e$ & $e$ & $\ldots$ & $e$ & $e$ & $\ldots$ & $e$ & $e$ & $e$ & $\ldots$ & $e$ & $e$ & $\ldots$ & $\ldots$ \\
\hline$s_{1} \in S_{e}$ & $e$ & $e$ & $e$ & $\ldots$ & $e$ & $e$ & $\ldots$ & $e$ & $e$ & $e$ & $\ldots$ & $e$ & $e$ & $\ldots$ & $\ldots$ \\
\hline$s_{2} \in S_{e}$ & $e$ & $e$ & $e$ & $\cdots$ & $e$ & $e$ & $\ldots$ & $e$ & $e$ & $e$ & $\cdots$ & $e$ & $e$ & $\cdots$ & $\cdots$ \\
\hline : & : & : & : & & : & $:$ & & 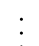 & : & : & & : & : & & \\
\hline$a_{1} \notin S_{e}$ & $e$ & $e$ & $e$ & $\ldots$ & $e$ & $e$ & $\ldots$ & $s_{i}$ & $s_{i}$ & $s_{i}$ & $\ldots$ & $s_{i}$ & $s_{i}$ & $\ldots$ & $\ldots$ \\
\hline$a_{2} \notin S_{e}$ & $e$ & $e$ & $e$ & $\ldots$ & $e$ & $e$ & $\ldots$ & $s_{j}$ & $s_{j}$ & $s_{j}$ & $\ldots$ & $s_{j}$ & $s_{j}$ & $\cdots$ & $\ldots$ \\
\hline$\vdots$ & : & : & : & & $\vdots$ & $:$ & & : & $:$ & : & & $\vdots$ & $:$ & & \\
\hline$t_{0}=f$ & $f$ & $f$ & $f$ & $\ldots$ & $f$ & $f$ & $\ldots$ & $f$ & $f$ & $f$ & $\ldots$ & $f$ & $f$ & $\ldots$ & $\ldots$ \\
\hline$t_{1} \in S_{f}$ & $f$ & $f$ & $f$ & $\ldots$ & $f$ & $f$ & $\ldots$ & $f$ & $f$ & $f$ & $\ldots$ & $f$ & $f$ & $\cdots$ & $\ldots$ \\
\hline$t_{2} \in S_{f}$ & $f$ & $f$ & $f$ & $\ldots$ & $f$ & $f$ & $\ldots$ & $f$ & $f$ & $f$ & $\ldots$ & $f$ & $f$ & $\ldots$ & $\ldots$ \\
\hline : & : & : & : & & : & $\vdots$ & & : & : & $\vdots$ & & $\vdots$ & $\vdots$ & & \\
\hline$b_{1} \notin S_{f}$ & $t_{k}$ & $t_{k}$ & $t_{k}$ & $\ldots$ & $t_{k}$ & $t_{k}$ & $\ldots$ & J & $f$ & $f$ & $\ldots$ & $f$ & $f$ & $\ldots$ & $\cdots$ \\
\hline$b_{2} \notin S_{f}$ & $t_{l}$ & $t_{l}$ & $t_{l}$ & $\ldots$ & $t_{l}$ & $t_{l}$ & $\ldots$ & $f$ & $f$ & $f$ & $\ldots$ & $f$ & $f$ & $\cdots$ & $\ldots$ \\
\hline : & : & : & : & & : & : & & . & : & : & & : & : & & \\
\hline & & & & & & & & & & & & & & & \\
\hline & $\vdots$ & $\vdots$ & $\vdots$ & & $\cdot$ & & & & $\vdots$ & $\cdot$ & & $\vdots$ & & & \\
\hline
\end{tabular}

\begin{tabular}{|c|c|c|c|c|c|c|c|c|c|c|c|c|c|c|c|}
\hline$L_{3}$ & $e$ & $s_{1}$ & $s_{2}$ & $\ldots$ & $a_{1}$ & $a_{2}$ & $\ldots$ & $f$ & $t_{1}$ & $t_{2}$ & $\ldots$ & $b_{1}$ & $b_{2}$ & $\ldots$ & . \\
\hline$s_{0}=e$ & $e$ & $e$ & $e$ & $\cdots$ & $e$ & $e$ & $\ldots$ & $e$ & $e$ & $e$ & $\cdots$ & $e$ & $e$ & $\ldots$ & $\ldots$ \\
\hline$s_{1} \in S_{e}$ & $e$ & $e$ & $e$ & $\ldots$ & $e$ & $e$ & $\ldots$ & $e$ & $e$ & $e$ & $\ldots$ & $e$ & $e$ & $\ldots$ & $\ldots$ \\
\hline$s_{2} \in S_{e}$ & $e$ & $e$ & $e$ & $\ldots$ & $e$ & $e$ & $\ldots$ & $e$ & $e$ & $e$ & $\ldots$ & $e$ & $e$ & $\ldots$ & $\ldots$ \\
\hline : & : & : & : & & : & : & & : & : & 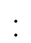 & & : & : & & \\
\hline$:$ & $\therefore$ & $:$ & $:$ & & $:$ & $:$ & & $:$ & : & : & & $:$ & $:$ & & \\
\hline$a_{1} \notin S_{e}$ & $e$ & $e$ & $e$ & $\cdots$ & $e$ & $\in E$ & $\cdots$ & $e$ & $e$ & $e$ & $\cdots$ & $\in E$ & $\in E$ & $\cdots$ & $\cdots$ \\
\hline$a_{2} \notin S_{e}$ & $e$ & $e$ & $e$ & $\cdots$ & $\in E$ & $e$ & $\ldots$ & $e$ & $e$ & $e$ & $\ldots$ & $\in E$ & $\in E$ & $\ldots$ & $\ldots$ \\
\hline & $\vdots$ & : & : & & $\vdots$ & $\vdots$ & & $\vdots$ & : & : & & $\vdots$ & $\vdots$ & & \\
\hline$t_{0}=f$ & $f$ & $f$ & $f$ & $\cdots$ & $f$ & $f$ & $\ldots$ & $f$ & $f$ & $f$ & $\cdots$ & $f$ & $f$ & $\ldots$ & $\cdots$ \\
\hline$t_{1} \in S_{f}$ & $f$ & $f$ & $f$ & $\cdots$ & $f$ & $f$ & $\ldots$ & $f$ & $f$ & $f$ & $\cdots$ & $f$ & $f$ & $\cdots$ & $\ldots$ \\
\hline$t_{2} \in S_{f}$ & $f$ & $f$ & $f$ & $\ldots$ & $f$ & $f$ & $\ldots$ & $f$ & $f$ & $f$ & $\ldots$ & $f$ & $f$ & $\ldots$ & $\ldots$ \\
\hline : & : & : & : & & : & : & & : & : & . & & : & : & & \\
\hline$b_{1} \notin S_{f}$ & $f$ & $f$ & $f$ & $\ldots$ & $\in E$ & $\in E$ & $\ldots$ & $f$ & $f$ & $f$ & $\ldots$ & $f$ & $\in E$ & $\ldots$ & $\ldots$ \\
\hline$b_{2} \notin S_{f}$ & $f$ & $f$ & $f$ & $\ldots$ & $\in E$ & $\in E$ & $\ldots$ & $f$ & $f$ & $f$ & $\ldots$ & $\in E$ & $f$ & $\ldots$ & $\ldots$ \\
\hline$\vdots$ & $\vdots$ & : & $\vdots$ & & $\vdots$ & $\vdots$ & & : & : & : & & : & $\vdots$ & & \\
\hline . & . & : & . & & . & . & & & . & . & & & . & & \\
\hline
\end{tabular}




\begin{tabular}{|c|c|c|c|c|c|c|c|c|c|c|c|c|c|c|c|}
\hline$L_{4}$ & $e$ & $s_{1}$ & $s_{2}$ & $\ldots$ & $a_{1}$ & $a_{2}$ & $\ldots$ & $f$ & $t_{1}$ & $t_{2}$ & $\ldots$ & $b_{1}$ & $b_{2}$ & $\ldots$ & $\ldots$ \\
\hline$s_{0}=e$ & $e$ & $e$ & $e$ & $\cdots$ & $e$ & $e$ & $\ldots$ & $e$ & $e$ & $e$ & $\ldots$ & $s_{i}$ & $s_{j}$ & $\ldots$ & $\ldots$ \\
\hline$s_{1} \in S_{e}$ & $e$ & $e$ & $e$ & $\cdots$ & $e$ & $e$ & $\cdots$ & $e$ & $e$ & $e$ & $\cdots$ & $s_{i}$ & $s_{j}$ & $\cdots$ & $\cdots$ \\
\hline$s_{2} \in S_{e}$ & $e$ & $e$ & $e$ & $\ldots$ & $e$ & $e$ & $\ldots$ & $e$ & $e$ & $e$ & $\ldots$ & $s_{i}$ & $s_{j}$ & $\ldots$ & $\ldots$ \\
\hline : & $\vdots$ & $\vdots$ & $\vdots$ & & $\vdots$ & $\vdots$ & & $\vdots$ & $\vdots$ & $\vdots$ & & : & $\vdots$ & & \\
\hline$a_{1} \notin S_{e}$ & $e$ & $e$ & $e$ & $\ldots$ & $e$ & $e$ & $\ldots$ & $e$ & $e$ & $e$ & $\ldots$ & $s_{i}$ & $s_{j}$ & $\cdots$ & $\ldots$ \\
\hline$a_{2} \notin S_{e}$ & $e$ & $e$ & $e$ & $\ldots$ & $e$ & $e$ & $\cdots$ & $e$ & $e$ & $e$ & $\ldots$ & $s_{i}$ & $s_{j}$ & $\cdots$ & $\ldots$ \\
\hline : & $\vdots$ & : & : & & : & : & & : & $\vdots$ & $\vdots$ & & : & : & & \\
\hline$t_{0}=f$ & $f$ & $f$ & $f$ & $\cdots$ & $t_{k}$ & $t_{l}$ & $\cdots$ & $f$ & $J$ & $f$ & $\ldots$ & $f$ & $f$ & $\cdots$ & $\ldots$ \\
\hline$t_{1} \in S_{f}$ & $f$ & $f$ & $f$ & $\ldots$ & $t_{k}$ & $t_{l}$ & $\ldots$ & $f$ & $f$ & $f$ & $\ldots$ & $f$ & $f$ & $\ldots$ & $\ldots$ \\
\hline$t_{2} \in S_{f}$ & $f$ & $f$ & $f$ & $\ldots$ & $t_{k}$ & $t_{l}$ & $\ldots$ & $f$ & $J$ & $f$ & $\ldots$ & $f$ & $f$ & $\ldots$ & $\ldots$ \\
\hline$\vdots$ & $\vdots$ & : & : & & $:$ & : & & : & : & $:$ & & $:$ & $:$ & & \\
\hline$b_{1} \notin S_{f}$ & $f$ & $f$ & $f$ & $\ldots$ & $t_{k}$ & $t_{l}$ & $\ldots$ & $f$ & J & $f$ & $\ldots$ & $f$ & $f$ & $\ldots$ & $\ldots$ \\
\hline$b_{2} \notin S_{f}$ & $f$ & $f$ & $f$ & $\cdots$ & $t_{k}$ & $t_{l}$ & $\cdots$ & $f$ & $J$ & $f$ & $\ldots$ & $f$ & $f$ & $\cdots$ & $\ldots$ \\
\hline : & $\vdots$ & $\vdots$ & $\vdots$ & & : & : & & : & : & : & & : & : & & \\
\hline & : & . & $\cdot$ & & $\cdot$ & $\cdot$ & & & : & $\vdots$ & & $:$ & . & & \\
\hline
\end{tabular}

\begin{tabular}{c||ccccccc|ccccccc|c}
$L_{5}$ & $e$ & $s_{1}$ & $s_{2}$ & $\ldots$ & $a_{1}$ & $a_{2}$ & $\ldots$ & $f$ & $t_{1}$ & $t_{2}$ & $\ldots$ & $b_{1}$ & $b_{2}$ & $\ldots$ & $\ldots$ \\
\hline \hline$s_{0}=e$ & $e$ & $e$ & $e$ & $\ldots$ & $e$ & $e$ & $\ldots$ & $e$ & $e$ & $e$ & $\ldots$ & $e$ & $e$ & $\ldots$ & $\ldots$ \\
$s_{1} \in S_{e}$ & $s_{1}$ & $s_{1}$ & $s_{1}$ & $\ldots$ & $s_{1}$ & $s_{1}$ & $\ldots$ & $s_{1}$ & $s_{1}$ & $s_{1}$ & $\ldots$ & $s_{1}$ & $s_{1}$ & $\ldots$ & $\ldots$ \\
$s_{2} \in S_{e}$ & $s_{2}$ & $s_{2}$ & $s_{2}$ & $\ldots$ & $s_{2}$ & $s_{2}$ & $\ldots$ & $s_{2}$ & $s_{2}$ & $s_{2}$ & $\ldots$ & $s_{2}$ & $s_{2}$ & $\ldots$ & $\ldots$ \\
$\vdots$ & $\vdots$ & $\vdots$ & $\vdots$ & & $\vdots$ & $\vdots$ & & $\vdots$ & $\vdots$ & $\vdots$ & & $\vdots$ & $\vdots$ & & \\
$a_{1} \notin S_{e}$ & $s_{i_{1}}$ & $s_{i_{1}}$ & $s_{i_{1}}$ & $\ldots$ & $s_{i_{1}}$ & $s_{i_{1}}$ & $\ldots$ & $s_{j_{1}}$ & $s_{j_{1}}$ & $s_{j_{1}}$ & $\ldots$ & $s_{j_{1}}$ & $s_{j_{1}}$ & $\ldots$ & $\ldots$ \\
$a_{2} \notin S_{e}$ & $s_{i_{2}}$ & $s_{i_{2}}$ & $s_{i_{2}}$ & $\ldots$ & $s_{i_{2}}$ & $s_{i_{2}}$ & $\ldots$ & $s_{j_{2}}$ & $s_{j_{2}}$ & $s_{j_{2}}$ & $\ldots$ & $s_{j_{2}}$ & $s_{j_{2}}$ & $\ldots$ & $\ldots$ \\
$\vdots$ & $\vdots$ & $\vdots$ & $\vdots$ & & $\vdots$ & $\vdots$ & & $\vdots$ & $\vdots$ & $\vdots$ & & $\vdots$ & $\vdots$ & & \\
\hline$t_{0}=f$ & $f$ & $f$ & $f$ & $\ldots$ & $f$ & $f$ & $\ldots$ & $f$ & $f$ & $f$ & $\ldots$ & $f$ & $f$ & $\ldots$ & $\ldots$ \\
$t_{1} \in S_{f}$ & $t_{1}$ & $t_{1}$ & $t_{1}$ & $\ldots$ & $t_{1}$ & $t_{1}$ & $\ldots$ & $t_{1}$ & $t_{1}$ & $t_{1}$ & $\ldots$ & $t_{1}$ & $t_{1}$ & $\ldots$ & $\ldots$ \\
$t_{2} \in S_{f}$ & $t_{2}$ & $t_{2}$ & $t_{2}$ & $\ldots$ & $t_{2}$ & $t_{2}$ & $\ldots$ & $t_{2}$ & $t_{2}$ & $t_{2}$ & $\ldots$ & $t_{2}$ & $t_{2}$ & $\ldots$ & $\ldots$ \\
$\vdots$ & $\vdots$ & $\vdots$ & $\vdots$ & & $\vdots$ & $\vdots$ & & $\vdots$ & $\vdots$ & $\vdots$ & & $\vdots$ & $\vdots$ & & \\
$b_{1} \notin S_{f}$ & $t_{k_{1}}$ & $t_{k_{1}}$ & $t_{k_{1}}$ & $\ldots$ & $t_{k_{1}}$ & $t_{k_{1}}$ & $\ldots$ & $t_{l_{1}}$ & $t_{l_{1}}$ & $t_{l_{1}}$ & $\ldots$ & $t_{l_{1}}$ & $t_{l_{1}}$ & $\ldots$ & $\ldots$ \\
$b_{2} \notin S_{f}$ & $t_{k_{2}}$ & $t_{k_{2}}$ & $t_{k_{2}}$ & $\ldots$ & $t_{k_{2}}$ & $t_{k_{2}}$ & $\ldots$ & $t_{l_{2}}$ & $t_{l_{2}}$ & $t_{l_{2}}$ & $\ldots$ & $t_{l_{2}}$ & $t_{l_{2}}$ & $\ldots$ & $\ldots$ \\
$\vdots$ & $\vdots$ & $\vdots$ & $\vdots$ & & $\vdots$ & $\vdots$ & & $\vdots$ & $\vdots$ & $\vdots$ & & $\vdots$ & $\vdots$ & & \\
\hline$\vdots$ & $\vdots$ & $\vdots$ & $\vdots$ & & $\vdots$ & $\vdots$ & & $\vdots$ & $\vdots$ & $\vdots$ & & $\vdots$ & $\vdots$ & &
\end{tabular}




\begin{tabular}{|c|c|c|c|c|c|c|c|c|c|c|c|c|c|c|c|}
\hline$L_{6}$ & $e$ & $s_{1}$ & $s_{2}$ & $\ldots$ & $a_{1}$ & $a_{2}$ & $\ldots$ & $f$ & $t_{1}$ & $t_{2}$ & $\ldots$ & $b_{1}$ & $b_{2}$ & $\ldots$ & $\ldots$ \\
\hline$s_{0}=e$ & $s_{i}$ & $s_{i}$ & $s_{i}$ & $\cdots$ & $s_{i}$ & $s_{i}$ & $\ldots$ & $s_{j}$ & $s_{j}$ & $s_{j}$ & $\cdots$ & $s_{j}$ & $s_{j}$ & $\cdots$ & $\cdots$ \\
\hline$s_{1} \in S_{e}$ & $s_{i}$ & $s_{i}$ & $s_{i}$ & $\cdots$ & $s_{i}$ & $s_{i}$ & $\cdots$ & $s_{j}$ & $s_{j}$ & $s_{j}$ & $\cdots$ & $s_{j}$ & $s_{j}$ & $\cdots$ & $\ldots$ \\
\hline$s_{2} \in S_{e}$ & $s_{i}$ & $s_{i}$ & $s_{i}$ & $\ldots$ & $s_{i}$ & $s_{i}$ & $\ldots$ & $s_{j}$ & $s_{j}$ & $s_{j}$ & $\ldots$ & $s_{j}$ & $s_{j}$ & $\cdots$ & $\ldots$ \\
\hline & . & . & . & & . & . & & . & . & . & & . & 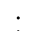 & & \\
\hline$:$ & : & : & : & & : & : & & : & : & : & & $:$ & $:$ & & \\
\hline$a_{1} \notin S_{e}$ & $s_{i}$ & $s_{i}$ & $s_{i}$ & $\cdots$ & $s_{i}$ & $s_{i}$ & $\ldots$ & $s_{j}$ & $s_{j}$ & $s_{j}$ & $\ldots$ & $s_{j}$ & $s_{j}$ & $\cdots$ & $\cdots$ \\
\hline$a_{2} \notin S_{e}$ & $s_{i}$ & $s_{i}$ & $s_{i}$ & $\ldots$ & $s_{i}$ & $s_{i}$ & $\cdots$ & $s_{j}$ & $s_{j}$ & $s_{j}$ & $\ldots$ & $s_{j}$ & $s_{j}$ & $\cdots$ & $\cdots$ \\
\hline & . & . & $\cdot$ & & . & . & & . & . & . & & - & . & & \\
\hline & : & $:$ & $:$ & & : & $:$ & & : & : & $:$ & & $:$ & $:$ & & \\
\hline$t_{0}=f$ & $t_{k}$ & $t_{k}$ & $t_{k}$ & $\ldots$ & $t_{k}$ & $t_{k}$ & $\ldots$ & $t_{l}$ & $t_{l}$ & $t_{l}$ & $\ldots$ & $t_{l}$ & $t_{l}$ & $\ldots$ & $\ldots$ \\
\hline$t_{1} \in S_{f}$ & $t_{k}$ & $t_{k}$ & $t_{k}$ & $\ldots$ & $t_{k}$ & $t_{k}$ & $\ldots$ & $t_{l}$ & $t_{l}$ & $t_{l}$ & $\ldots$ & $t_{l}$ & $t_{l}$ & $\ldots$ & $\ldots$ \\
\hline$t_{2} \in S_{f}$ & $t_{k}$ & $t_{k}$ & $t_{k}$ & $\ldots$ & $t_{k}$ & $t_{k}$ & $\ldots$ & $t_{l}$ & $t_{l}$ & $t_{l}$ & $\ldots$ & $t_{l}$ & $t_{l}$ & $\ldots$ & $\ldots$ \\
\hline & . & $\cdot$ & 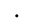 & & . & $\cdot$ & & . & $\cdot$ & 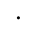 & & 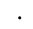 & $\cdot$ & & \\
\hline : & : & $:$ & : & & $\cdot$ & : & & : & $\cdot$ & $:$ & & : & $:$ & & \\
\hline$b_{1} \notin S_{f}$ & $t_{k}$ & $t_{k}$ & $t_{k}$ & $\cdots$ & $t_{k}$ & $t_{k}$ & . & $t_{l}$ & $t_{l}$ & $t_{l}$ & $\ldots$ & $t_{l}$ & $t_{l}$ & $\cdots$ & $\cdots$ \\
\hline$b_{2} \notin S_{f}$ & $t_{k}$ & $t_{k}$ & $t_{k}$ & $\ldots$ & $t_{k}$ & $t_{k}$ & $\cdots$ & $t_{l}$ & $t_{l}$ & $t_{l}$ & $\ldots$ & $t_{l}$ & $t_{l}$ & $\ldots$ & $\ldots$ \\
\hline . & 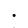 & 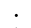 & 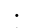 & & • & . & & . & . & . & & 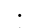 & . & & \\
\hline$:$ & : & $:$ & : & & $:$ & : & & $\cdot$ & 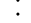 & $:$ & & : & $:$ & & \\
\hline . & . & : & $\cdot$ & & : & . & & . & . & $\cdot$ & & $\cdot$ & · & & \\
\hline${ }^{*}$ & $:$ & : & $:$ & & $:$ & $:$ & & : & $:$ & $:$ & & $:$ & $:$ & & \\
\hline
\end{tabular}

\section{References}

1. Atre, A.K.: On a semigroup which is an inflation of its set of factorizable elements. Vignana Bharathi 1, 69-72 (1975)

2. Boltnev, A.A.: A set-theoretic description of semigroups of some varieties. Chebyshevskiı̌ Sb. 6, 56-63 (2005). (in Russian)

3. Evans, T.: Products of points—-some simple algebras and their identities. Am. Math. Mon. 74, 362372 (1967)

4. Lehtonen, E., Waldhauser, T.: Minor posets of functions as quotients of partition lattices. Order 36, 23-41 (2019)

5. Machida, H., Rosenberg, I.G.: Essentially minimal clones of rank 3 on a three-element set. In: Drechsler, R., Wille, R. (eds.) 44th International Symposium on Multiple-Valued Logic (ISMVL 2014), pp. 97-102. IEEE Computer Society, Los Alamitos, CA (2014)

6. Machida, H., Waldhauser, T.: Lazy clones and essentially minimal groupoids. In: Gaudet, V.C., Dueck, G.W. (eds.) 45th International Symposium on Multiple-Valued Logic (ISMVL 2015), pp. 199-204. IEEE Computer Society, Los Alamitos, CA (2015)

7. Płonka, J.: Diagonal algebras. Fund. Math. 58, 309-321 (1966)

Publisher's Note Springer Nature remains neutral with regard to jurisdictional claims in published maps and institutional affiliations. 